\title{
Modeling and Optimization of an Electrostatic Energy Harvesting Device
}

\section{Crovetto, Andrea; Wang, Fei; Hansen, Ole}

\section{Published in:}

I E E E Journal of Microelectromechanical Systems

Link to article, DOI:

10.1109/JMEMS.2014.2306963

Publication date:

2014

Link back to DTU Orbit

\section{Citation (APA):}

Crovetto, A., Wang, F., \& Hansen, O. (2014). Modeling and Optimization of an Electrostatic Energy Harvesting Device. I E E E Journal of Microelectromechanical Systems, 23(5), 1141-1155.

https://doi.org/10.1109/JMEMS.2014.2306963

\section{General rights}

Copyright and moral rights for the publications made accessible in the public portal are retained by the authors and/or other copyright owners and it is a condition of accessing publications that users recognise and abide by the legal requirements associated with these rights.

- Users may download and print one copy of any publication from the public portal for the purpose of private study or research.

- You may not further distribute the material or use it for any profit-making activity or commercial gain

- You may freely distribute the URL identifying the publication in the public portal 


\title{
Modeling and Optimization of an Electrostatic Energy Harvesting Device
}

\author{
Andrea Crovetto, Fei Wang, Senior Member, IEEE, and Ole Hansen
}

\begin{abstract}
Modeling of energy harvesting devices is complicated by the coupling between electrical and mechanical domains. In this paper, we present a coupled electromechanical model for electret-based resonant energy harvesters where the two output pads are placed on the same device side (single-sided). An analytical analysis is complemented by 2-D finite element method simulations, where the fringing field effect on a plane capacitor is studied and accounted for by an effective area that is well fitted by a sinusoidal function of the displacement of the proof mass. From analytical calculations, we prove that the electrostatic transducer force is related to the voltage output and cannot be approximated by viscous damping or a Coulomb force as reported previously. The coupled model with two simultaneous differential equations is numerically solved for the voltage output and transduction force with given parameters. The model was verified both by practical measurements from our own fabricated device and results from a reference. An optimization study is carried out using this model to achieve the maximum output power by tuning the allowable movement $\left(X_{M}\right)$ of the proof mass. Finally, the effect of a standard power-conditioning circuit is investigated for both continuous and burst power supply applications.

[2013-0207]
\end{abstract}

Index Terms-Energy harvesting, MEMS, electret, modeling.

\section{INTRODUCTION}

$\mathbf{T}$ HANKS to the advanced technology of micro-electromechanical systems (MEMS), many wireless sensors have been developed recently and the cost is decreasing with the continuous down scaling of chip size. These sensor nodes typically demand low power cost and their performance will be greatly enhanced when the battery is eliminated and they

Manuscript received June 29, 2013; revised November 15, 2013; accepted February 2, 2014. This work was supported in part by the Danish Research Council for Technology and Production under Project 10-080864, in part by the Danish National Research Foundation's Center for Individual Nanoparticle Functionality under Grant CINF DNRF54, and in part by the Start Grant from the South University of Science and Technology of China. Subject Editor R. Ghodssi. (Corresponding author: F. Wang.)

A. Crovetto was with Politecnico di Milano, Milan 20133, Italy. He is now with the Department of Micro- and Nanotechnology, Technical University of Denmark, DTU Nanotech, Kgs. Lyngby DK 2800, Denmark (e-mail: crovetto.andrea@gmail.com).

F. Wang was with the Department of Micro- and Nanotechnology, Technical University of Denmark, DTU Nanotech, Kgs. Lyngby DK-2800, Denmark. $\mathrm{He}$ is now with the Department of Electronic and Electrical Engineering, South University of Science and Technology of China, Shenzhen 518055, China (e-mail: wang.f@ sustc.edu.cn).

O. Hansen is with the Department of Micro- and Nanotechnology, Technical University of Denmark, DTU Nanotech, Kgs. Lyngby DK-2800, Denmark, and also with the Center for Individual Nanoparticle Functionality, Technical University of Denmark, DTU Nanotech, Kgs. Lyngby DK-2800, Denmark (e-mail: ole.hansen@nanotech.dtu.dk).

Color versions of one or more of the figures in this paper are available online at http://ieeexplore.iee.org.

Digital Object Identifier 10.1109/JMEMS.2014.2306963 (a)

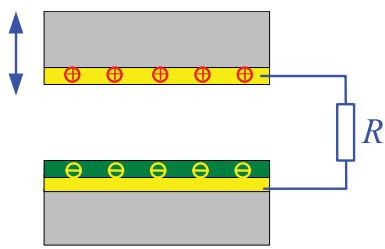

(b)

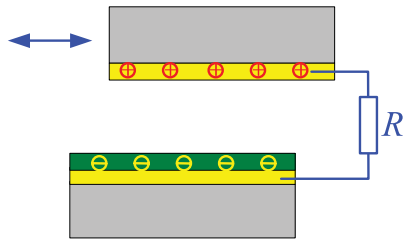

(c)
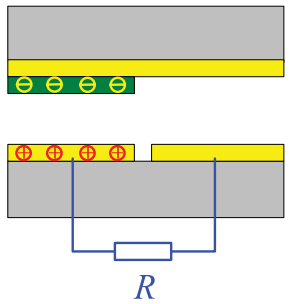

Fig. 1. Three configurations of the electret based energy harvesting devices using variable capacitors by (a) changing the gap; (b) changing the overlap area; (c) changing the counter electrodes.

become self-powered, for example, by harvesting energy from the environment [1], [2]. A number of vibrational energy harvesting devices have been fabricated during the past decade based on piezoelectric [3], [4], electromagnetic [5], [6] and electrostatic [7]-[15] transduction methods. Among these, the electrostatic systems have advantages of both compatibility with MEMS processes and small size. Using variable capacitors, induced charges from an external voltage bias [8] or pre-charged [9]-[15] electrets can move back and forth through an external load and power is generated when proof mass structure resonates according to the vibration source.

Fig. 1 shows three different configurations of variable capacitors with air gap for energy harvesting. With out-of-plane or in-plane vibration sources, the capacitances vary by changing the gap distance in Fig. 1(a) or by changing the overlap area in Fig. 1(b). A few electret generators have been developed based on these two schemes [9], [10], [16]. Simulation and calculation have been made based on Boland's model which was initially proposed for a rotational generator [17]. This model has been incorporated by Boisseau et al. [18] into a 
complete electromechanical model and used in a parameter optimization study.

In these two schemes, the external load is connected between the two electrodes on both sides (EBS) of the devices. However, as research progresses from simple proof-of-concept prototypes to fully packaged devices, there are some technical challenges for the EBS design. Thin metal signal lines on the beam structures have to withstand the bending stress during the vibration. With a device lifetime of 5 years and a working frequency of $100 \mathrm{~Hz}$, the fatigue limit of the metal lines should be larger than $10^{10}$ cycles. Moreover, it is typically difficult to lead out the signal lines from both sides of the device, where additional wiring or 3D structures such as metal stud bumps are demanded [19]. Therefore, placing the two output electrodes on a single side (ESS) of the same substrate becomes the design approach of choice, as shown in Fig. 1(c). The metal signal lines stay static during the vibration and the signal lead outs are fabricated easily by a simple metal patterning using photolithography. This ESS scheme has been proved to be promising both in prototype devices [12], [13] and MEMS devices fabricated at wafer-scale [14].

Boland's model is unfortunately not applicable to the ESS design because the base electrode is now electrically isolated and the three independent electrodes in Fig. 1(c) are at different potentials. A completely different modeling approach, applicable to a variety of electromechanical transduction methods, was proposed by Williams and Yates [20] and later refined by Mitcheson et al. [21]. The transducer damping factor $\xi_{\mathrm{t}}$ and air damping factor $\xi_{\mathrm{c}}$ were introduced to describe the effect of power extraction and power loss on air friction. The optimal damping factor can be calculated from a few design parameters, source vibration features and the condition of equal damping factors $\left(\xi_{\mathrm{t}}=\xi_{\mathrm{c}}\right)$, resulting in the following maximum generated power at resonance:

$$
P_{\text {res }}=\frac{1}{2} m Y_{0}^{2} \omega^{3} \frac{X_{\mathrm{M}}}{Y_{0}} .
$$

Here $Y_{0}$ and $\omega$ are the amplitude and angular frequency of the vibration source; $m$ and $X_{\mathrm{M}}$ are the mass and maximum displacement limit of the harvester proof mass. The optimal $\xi_{\mathrm{t}}$ allows the proof mass to vibrate at an amplitude of $X_{\mathrm{M}}$.

This general treatment is potentially applicable to electromagnetic, piezoelectric, and electrostatic devices because all the parameters that are specific to just one transduction method are included in the damping factor $\xi_{\mathrm{t}}$. However, some issues may arise when this model is applied to any of the three electret-based setups in Fig. 1. First of all, there is no guarantee that the optimal $\xi_{\mathrm{t}}$ is technically feasible or that it will yield a usable output signal, especially regarding the optimal load. Even more important, the damping factor $\xi_{\mathrm{t}}$ is introduced under the assumption that transducer damping is equivalent to a viscous force on the proof mass

$$
F_{v}(t)=-D \dot{x}(t)=-2 m \omega_{0} \xi_{\mathrm{t}} \dot{x}(t),
$$

where $D$ is a positive constant, $\dot{x}(t)$ is the proof mass velocity and $\omega_{0}$ its resonance frequency. Mitcheson et al. [21] have also covered the case of a Coulomb-type transducer damping force

$$
F \mathrm{c}(t)=-C \operatorname{Sign}[\dot{x}(t)],
$$

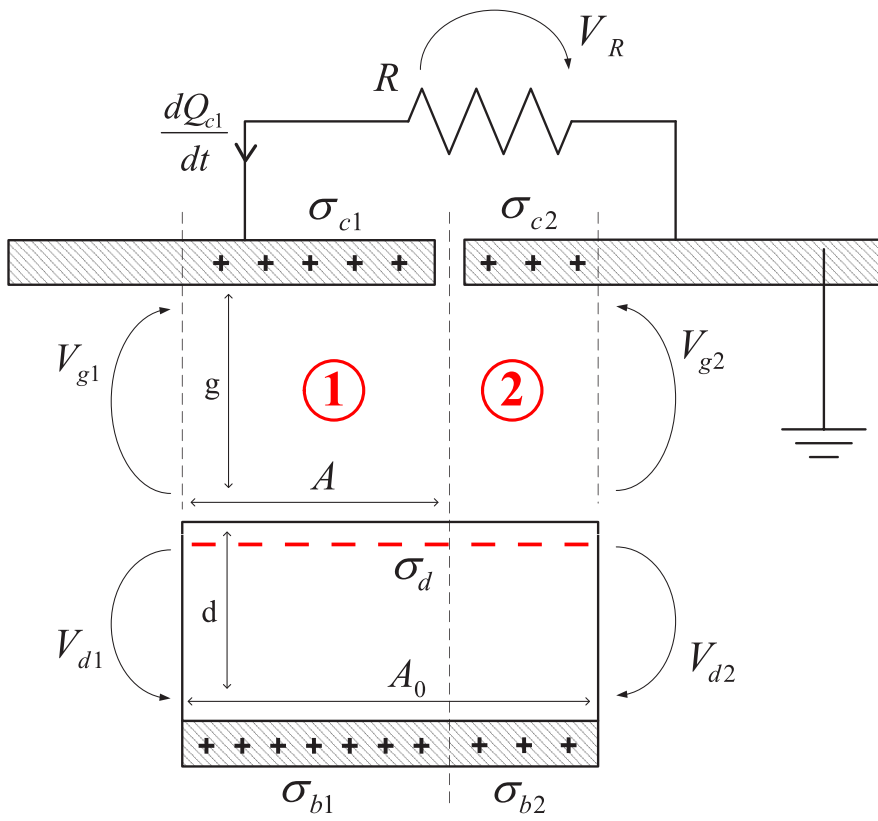

Fig. 2. Sketch of the electrical domain for the electrodes on single-side (ESS) harvester design.

(where $C$ is a positive constant), showing that under optimal damping conditions the maximum generated power at resonance is still given by Eq. 1. However, both proposed force expressions were originally introduced to model other types of energy harvesters: the viscous force for electromagnetic generators and the Coulomb force for comb drives operated in constant voltage mode [21]. More accurate analysis of the electrostatic transduction force $F_{\mathrm{t}}$ is necessary for the ESS design. Therefore, it is our goal to derive the actual shape of the transducer force in ESS harvesters from both analytical expressions and numerical calculations.

Based on the above remarks, our work aims at (1) modifying Boland's electrical model as appropriate for the ESS design, (2) refining the plane capacitor model with an finite element method (FEM) electrostatic study to take fringing fields and multiple electrode overlaps into account, (3) coupling the electrical and mechanical domains as two simultaneous equations, (4) validating model predictions with available data from the literature, (5) evaluating the applicability of the viscous- or Coulomb-type transducer force approximations, and (6) showing how device parameters could be optimized to obtain maximum output power.

\section{Modified Electrical Model}

In the analysis we consider the single-sided harvester device shown schematically in Fig. 2, where a single period of a periodic arrangement of electret covered base electrodes and counter electrode pairs (electrodes 1 and 2) is shown. As illustrated the overlap area of electrode 1 and the base electrode is $A$ and it can reach a maximum value of $A_{0}$, which is the area of the electret covered electrode; the figure shows the situation where the base electrode is displaced from the ideal equilibrium position with equal overlap areas. The surface of the electret dielectric is charged to the uniform, 
permanent surface charge density $\sigma_{\mathrm{d}}$. The electret material has the relative permittivity $\varepsilon_{\mathrm{d}}$ and the thickness $d$, while the remaining separation of the base and counter electrodes is an air gap of thickness $g$ and absolute permittivity $\varepsilon_{0}$.

In the analysis we shall assume that the gap between the counter electrodes is small enough to be taken as zero. In the analytical analysis we shall also assume that a quasi one dimensional plate capacitor model is sufficiently accurate, and use finite element modeling (FEM) to analyze the resulting limitations and improve the final model.

\section{A. Plane Capacitor Approximation - Static System}

We will first discuss the situation where all metal electrodes in the system are at ground potential. Overall charge neutrality of the system then requires that $\sigma_{\mathrm{d}}+\sigma_{\mathrm{c}}+\sigma_{\mathrm{b}}=0$, where $\sigma_{\mathrm{c}}$, and $\sigma_{\mathrm{b}}$ are the charge densities in overlap areas on the counter and base electrodes, respectively. Grounding of the electrodes requires $V_{\mathrm{g}}=V_{\mathrm{d}}=\sigma_{\mathrm{c}} g / \varepsilon_{0}=\sigma_{\mathrm{b}} d /\left(\varepsilon_{\mathrm{d}} \varepsilon_{0}\right)$ where $V_{\mathrm{g}}$ is the potential difference between the counter electrodes and the electret surface; $V_{\mathrm{d}}$ is the potential difference between the base electrodes and the electret surface. It follows that the surface charge densities on the base and counter electrodes are

$$
\sigma_{\mathrm{b}}=-\sigma_{\mathrm{d}} \frac{\varepsilon_{\mathrm{d}} g}{d+g \varepsilon_{\mathrm{d}}},
$$

and

$$
\sigma_{\mathrm{c}}=-\sigma_{\mathrm{d}} \frac{d}{d+g \varepsilon_{\mathrm{d}}},
$$

respectively.

Grounding of the electrodes forces the system into an electrostatically well defined state, and if the system is at rest when grounding is done, the system state is fully defined. Even if deliberate grounding is not performed the real system will with time reach this state when left alone, since excess charge on the electrodes will leak to ground; the time to reach equilibrium can, however, be quite long and a brief grounding of the electrodes after assembly prior to operation is desirable.

\section{B. Plane Capacitor Approximation - Vibrating System}

When grounding of the electrodes is lifted, a load resistor $R$ is connected between electrodes 1 and 2, and the system is allowed to vibrate. The electrode potentials and electrode surface charge densities become time dependent quantities depending on the instantaneous state of the system, and at most one electrode (e.g. electrode 2) is at ground reference potential. Note, however, that the electret surface charge density $\sigma_{\mathrm{d}}$ remains constant. The total charge $A_{0} \sigma_{\mathrm{b}}$ on the base electrode also remains constant (on the time scale relevant for the response to vibrations) since it is left floating; the distribution of the surface charge density on the base electrode, however, varies with time. We shall assume charge neutrality (complete charge induction) for the individual overlap areas and thus

$$
\sigma_{\mathrm{d}}+\sigma_{\mathrm{c} i}(t)+\sigma_{\mathrm{b} i}(t)=0,
$$

where $\sigma_{\mathrm{c} i}$ and $\sigma_{\mathrm{b} i}(i \in[1,2])$ are the surface charge densities in regions 1 and 2 on the counter and base electrodes, respectively. This equation governs the distribution of the charge on the base electrode when combined with the requirement of a constant total charge on the floating base electrode

$$
A_{0} \sigma_{\mathrm{b}}=A(t) \sigma_{\mathrm{b} 1}(t)+\left[A_{0}-A(t)\right] \sigma_{\mathrm{b} 2}(t)=\mathrm{constan} t,
$$

where $A_{0} \sigma_{\mathrm{b}}$ is the base electrode charge obtained during the initial grounding of the system electrodes, and $A(t)$ the instantaneous overlap area between electrode 1 and the base electrode.

The instantaneous charge on counter electrode 1 is $Q(t)=$ $A(t) \sigma_{\mathrm{c} 1}(t)$ as a result of the current flow through the load resistor. The voltage across the resistor $V_{\mathrm{R}}$ must therefore fulfil

$$
R \frac{\mathrm{d} Q(t)}{\mathrm{d} t}=V_{\mathrm{R}}(t)=V_{\mathrm{t}(t)}
$$

where $V_{\mathrm{t}(t)}=V_{\mathrm{g} 2}(t)-V_{\mathrm{d} 2}(t)+V_{\mathrm{d} 1}(t)-V_{\mathrm{g} 1}(t)$ is the total voltage between counter electrodes 1 and 2 (transducer voltage). The relation between the voltages is obtained from Kirchhoff's voltage law, see Fig. 2.

The potential difference between a counter electrode and the local electret surface is

$$
V_{\mathrm{g} i}(t)=\sigma_{\mathrm{c} i}(t) g / \varepsilon_{0},
$$

while the corresponding potential difference between the base electrode and the local electret surface is

$$
V_{\mathrm{d} i}(t)=\sigma_{\mathrm{b} i}(t) d /\left(\varepsilon_{\mathrm{d}} \varepsilon_{0}\right) .
$$

By use of Eqs. 4-10 the load equation, Eq. 8, may be rewritten in compact notation as

$$
\frac{\mathrm{d} Q}{\mathrm{~d} t}=\left(-\frac{d+g \varepsilon_{\mathrm{d}}}{R \varepsilon_{\mathrm{d}} \varepsilon_{0}} \frac{Q}{A}-\frac{d \sigma_{\mathrm{d}}}{R \varepsilon_{\mathrm{d}} \varepsilon_{0}}\right) \frac{A_{0}}{A_{0}-A} .
$$

If the factor $A_{0} /\left(A_{0}-A\right)$ is disregarded, Eq. 11 is precisely Boland's differential equation for the EBS harvesting system [17]. Due to the additional term it is not possible to solve it analytically, neither for a linear nor for a sinusoidal $A(t)$ pattern. However, a qualitative prediction can be made: because the term $A_{0} /\left(A_{0}-A\right)$ is always larger than 1 , the ESS configuration allows larger currents than that of the EBS configuration and thus a shorter response time is expected. To validate this hypothesis, Boland's equation and Eq. 11 are both solved numerically with the same geometric parameters $\left(d=g=10 \mu \mathrm{m}, \epsilon_{\mathrm{d}}=2, A_{0}=25 \mathrm{~mm}^{2}, \sigma_{\mathrm{d}}=0.35 \mathrm{mC} / \mathrm{m}^{2}\right)$ under a sample sinusoidal vibration $A(t)=A_{0} / 2+$ $\left(A_{0} / 3\right) \sin (2 \pi f t)$ with frequency $f=1 / T=200 \mathrm{~Hz}$. The boundary condition $Q(0)=Q(T)$ is set in order to account for periodicity in the charge function.

With a load resistance of $50 \mathrm{M} \Omega$ the two design types are almost indistinguishable [Fig. 3(a)]. At $500 \mathrm{M} \Omega$ load resistance, however, a significant differences arise [Fig. 3(b)]. First of all, there is a larger phase lag between the mechanical vibration $A(t)$ and the charge oscillations $Q(t)$ due to the loading effect that slows down the circuit. For that reason, the charge oscillation amplitudes are smaller than those at $R=50 \mathrm{M} \Omega$. This effect is more pronounced for the interchip case, which also has a slightly more deformed charge solution compared to a pure sine function. The optimal load 

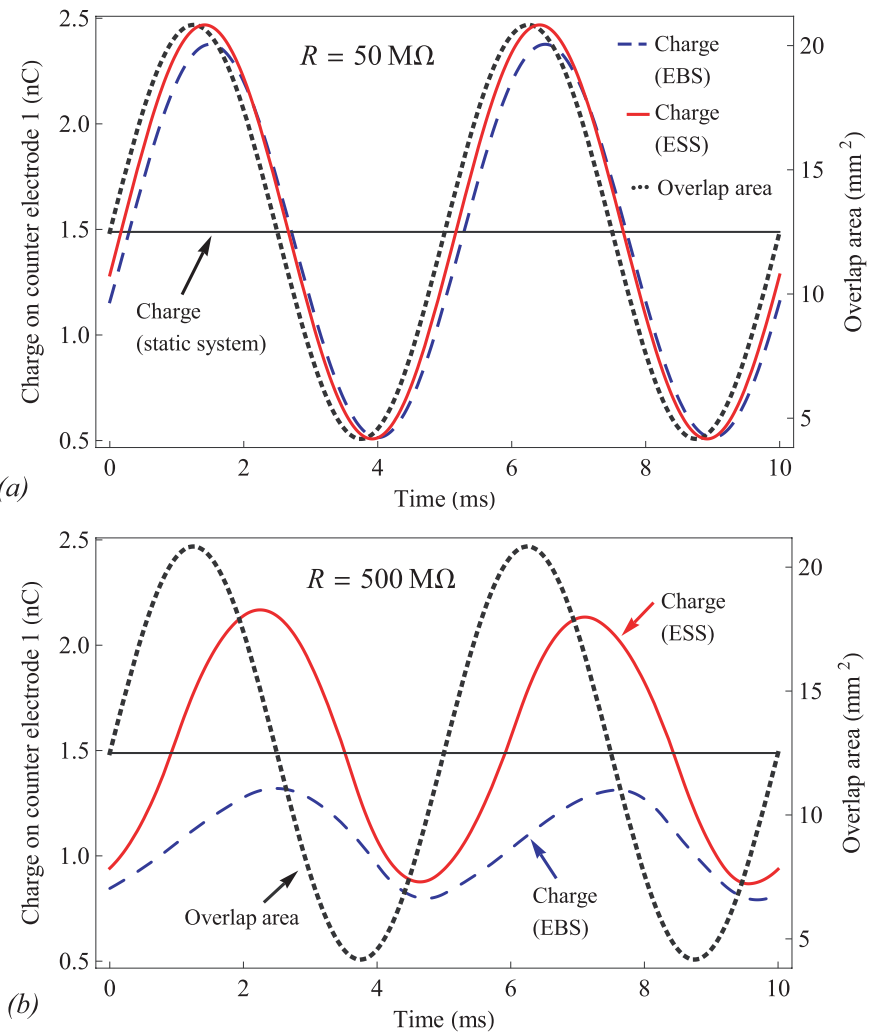

Fig. 3. Solutions of equation 11 (ESS design) and of Boland's equation (EBS design) under identical conditions. (a) $R=50 \mathrm{M} \Omega$. (b) $R=500 \mathrm{M} \Omega$.

for the EBS harvester can be calculated from the following formula [18]:

$$
R_{o p t}=\frac{d+\epsilon_{\mathrm{d}} g}{\epsilon_{0} \frac{d A}{d t}}
$$

Approximating $d A / d t$ with $\Delta A / T$ yields $R_{o p t} \simeq 508 \mathrm{M} \Omega$. Unless the load resistance is much lower than this value [as in Fig. 3(a)], the ESS design can generate more power at the same load due to its faster response time resulting in a higher $R_{o p t}$. With the data from Fig. 3(a), at $500 \mathrm{M} \Omega$ the ESS design is expected to extract $59.5 \mu \mathrm{W}$ versus $9.7 \mu \mathrm{W}$ for the EBS design.

\section{Fringing Fields}

There are three main limitations in the plane capacitor model. First, the plane capacitor approximation is accurate only when the gap $g$ is much smaller than the electret width $w$. Fringing fields induce charge on neighboring counter electrodes (Fig. 4), reducing the maximum obtainable charge difference between counter electrodes 1 and 2. Second, Eq. 11 fails at modeling the case with large vibration amplitude where the electret overpasses more than two counter electrodes during one period of oscillation. The terms $A(t)$ and $A_{0}-A(t)$ in the denominator will cause singularities when the electret is at full overlap with one counter electrode $\left(A=0\right.$ or $\left.A=A_{0}\right)$. Third, neighboring counter electrodes do have a small spacing between each other where charge is not induced.

To overcome the issues mentioned above, a 2D FEM study was carried out in Comsol Multiphysics, similarly to [18].

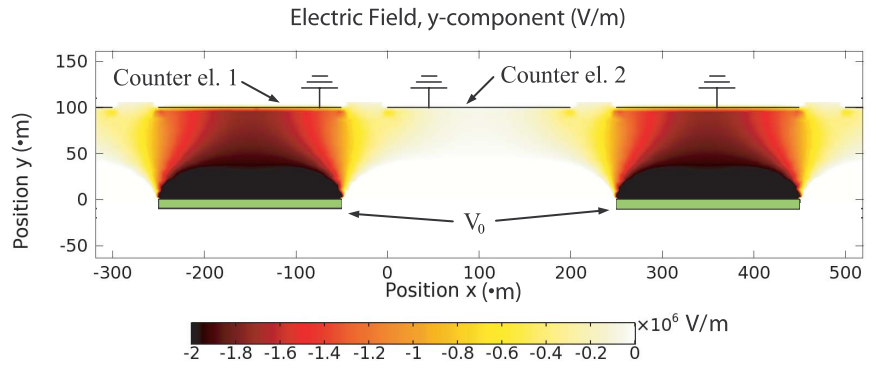

Fig. 4. FEM simulation of the exact electric field in the active region. The back side of the electret is set to a sample voltage $V_{0}$ and all counter electrodes are set to ground potential.

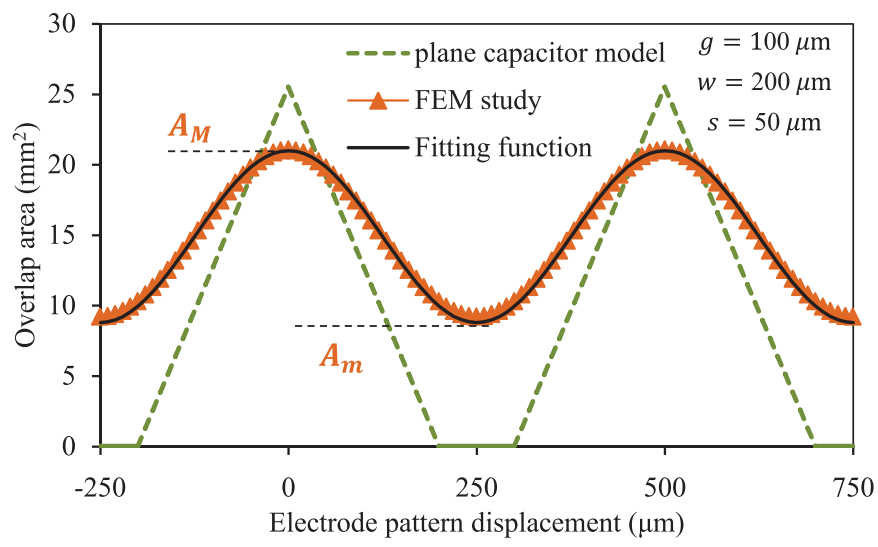

Fig. 5. Effective overlap area between the electret and counter electrode 1 as a function of their relative displacement. The function is spatially periodic with a $500 \mu \mathrm{m}$ pitch and can be approximated by a sine function. The overlap area obtained with a plane capacitor model is also shown for comparison.

The back side of the electret is defined with a voltage of $V_{0}$, while both counter electrodes are modeled as infinitely thin metal layers and kept at ground potential (Fig. 4). The electret width $w=200 \mu \mathrm{m}$ and the air gap $g=100 \mu \mathrm{m}$ are defined which means $w / g=2$. The spacing between counter electrodes is $s=50 \mu \mathrm{m}$. The parameters are those of a real device in [14] where the plane capacitor approximation shows its limitations. In the FEM study, the counter electrode pattern is swept horizontally in $10 \mu \mathrm{m}$ steps in order to sample the induction charge on counter electrode 1 as a function of pattern displacement $x$. At each step the charge on the counter electrode 1 is calculated and compared to the analytic expectation. This naturally leads to the definition of an 'effective' overlap area $\tilde{A}(x)=Q / \sigma_{c 1}$, which is plotted in Fig. 5. It is evident that $\tilde{A}(x)$ is much more resemblant of a sinusoidal function than of the triangular function predicted by the plane capacitor model. Therefore the fitting function

$$
\tilde{A}(x)=\frac{A_{\mathrm{M}}+A_{\mathrm{m}}}{2}+\left(\frac{A_{\mathrm{M}}-A_{\mathrm{m}}}{2}\right) \cos \left(\frac{\pi}{w+s} x+\phi\right)
$$

can be employed as an excellent approximation. $A_{\mathrm{M}}$ and $A_{\mathrm{m}}$ are the maximum and minimum values of the FEM-sampled overlap function (Fig. 5), respectively. The excess phase term $\phi$ depends on the initial pattern overlap (i.e. at $x=0$ ).

The unavoidable presence of counter electrode 2 at a small distance from counter electrode 1 results in a 
cross-talking effect: a significant amount of induction charge is observed even when the actual overlap with the electret is zero and the peak to peak charge amplitude is reduced. This phenomenon negatively affects output power and should be taken into account.

\section{Coupled Electromechanical Model}

A reasonable approximation for the equation of motion of the proof mass (mass $m$ ) is [22]:

$$
m \ddot{x}=-m \ddot{y}-k x-D \dot{x}+F_{\mathrm{t}} .
$$

Here $x$ is again the displacement between the two device sides, i.e. the proof mass relative displacement. $y$ is the displacement of the external vibration and hence $m \ddot{y}$ is the driving force exerted by the vibration source on the proof mass, $k x$ is a linear elastic force due to a spring structure of overall stiffness $k . D \dot{x}$ is a viscous damping force arising from the viscous mechanical resistance of the surrounding fluid. $F_{\mathrm{t}}$ is the transducer force and requires further investigation.

\section{A. Transducer Force}

For a conservative transducer in thermodynamic equilibrium, the energy put into the capacitors through the electric and mechanical ports is given by

$$
\mathrm{d} U_{e}=-V_{\mathrm{t}} \mathrm{d} Q_{\mathrm{t}}-F_{\mathrm{t}} \mathrm{d} x_{\mathrm{t}},
$$

where $U_{e}$ is the total electrostatic energy contained in the device, and $V_{\mathrm{t}}$ is the output voltage between the two electrodes 1 and 2. From Fig. 2, the total electrostatic energy in the device is

$$
\begin{aligned}
U_{e}= & \frac{1}{2} \frac{g A}{\varepsilon_{0}} \sigma_{c 1}^{2}+\frac{1}{2} \frac{g\left(A_{0}-A\right)}{\varepsilon_{0}} \sigma_{c 2}^{2} \\
& +\frac{1}{2} \frac{d A}{\varepsilon_{0} \varepsilon_{\mathrm{d}}} \sigma_{b 1}^{2}+\frac{1}{2} \frac{d\left(A_{0}-A\right)}{\varepsilon_{0} \varepsilon_{\mathrm{d}}} \sigma_{b 2}^{2} \\
= & \frac{1}{2} A_{0} \frac{g d}{\varepsilon_{0}\left(g \varepsilon_{\mathrm{d}}+d\right)} \sigma_{\mathrm{d}}^{2}+\frac{1}{2} \frac{A_{0}}{A\left(A_{0}-A\right)} \frac{g \varepsilon_{\mathrm{d}}+d}{\varepsilon_{0} \varepsilon_{\mathrm{d}}}\left(A \sigma_{\mathrm{c}}-Q\right)^{2} \\
= & U_{0}+\frac{1}{2} \frac{\left(Q-Q_{s c}\right)^{2}}{C},
\end{aligned}
$$

where the actual charge on electrode 1 is $Q=A \sigma_{c 1}$ and the short circuit charge on electrode 1 is $Q^{s c}=A \sigma_{\mathrm{c}}$. We see that the electrostatic energy has an invariant component $U_{0}$ that only depends on the transducer geometry and the electret charge density and a contribution that is an ordinary capacitive energy contribution, where the capacitance is

$$
C=\frac{A\left(A_{0}-A\right)}{A_{0}} \frac{\varepsilon_{0} \varepsilon_{\mathrm{d}}}{g \varepsilon_{\mathrm{d}}+d},
$$

as expected. The expression in Eq. 16 is generally true when we include the fringe effect with the effective area $\tilde{A}(x)$. With Eq. 15 and Eq. 16, we get the voltage

$$
V_{\mathrm{R}}=V_{\mathrm{t}}=-\frac{\partial U}{\partial Q}=-\frac{Q-Q^{s c}}{C}
$$

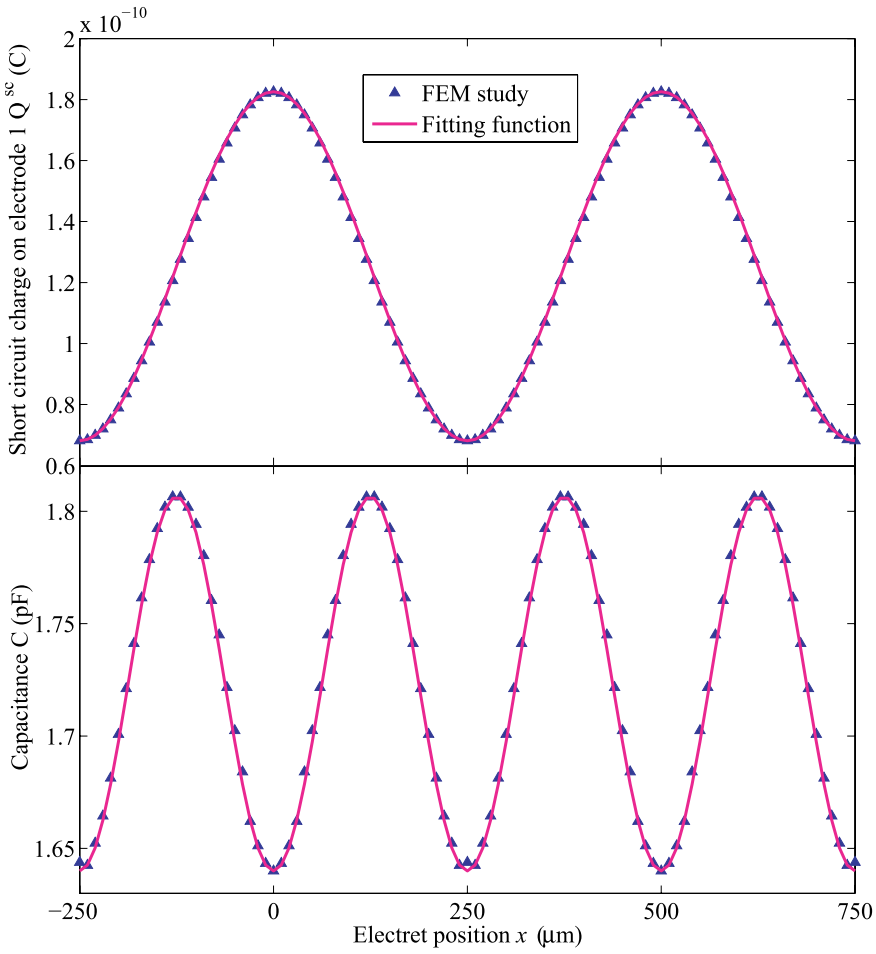

Fig. 6. FEM Simulation of the short circuit charge $Q^{s c}$ on electrode 1 and the capacitance $C$ of the device.

and the transduction force

$$
\begin{aligned}
F_{\mathrm{t}} & =-\frac{\partial U}{\partial x} \\
& =-\left(-\frac{Q-Q^{s c}}{C} \frac{\mathrm{d} Q^{s c}}{\mathrm{~d} x}-\frac{1}{2} \frac{\left(Q-Q^{s c}\right)^{2}}{C^{2}} \frac{\mathrm{d} C}{\mathrm{~d} x}\right) \\
& =-\left(V_{\mathrm{t}} \frac{\mathrm{d} Q^{s c}}{\mathrm{~d} x}-\frac{1}{2} V_{\mathrm{t}}^{2} \frac{\mathrm{d} C}{\mathrm{~d} x}\right),
\end{aligned}
$$

The dependence of $Q^{s c}=Q^{s c}(x)$ and $C=C(x)$ on the electret position $x$ are studied in a more detailed FEM simulation. As shown in Fig. 6, both the short circuit charge and the device capacitance may also be well fitted by sinusoidal functions. The short circuit charge is seen to vary with the spatial frequency of the electrode structure and has a significant amplitude, while the capacitance varies with twice the spatial frequency of the electrode structure and with a very small amplitude. It can be seen from the simulation that the first term in Eq. 18 is dominant when the voltage is low.

\section{B. Full System}

Equations 11 and 14 can now be solved simultaneously as a set of coupled differential equations with variables $x$ and $Q$. The equation set is

$$
\left\{\begin{array}{l}
\ddot{x}=a_{0} \sin (\omega t)-\omega_{0}^{2} x-2 \xi_{\mathrm{m}} \omega_{0} \dot{x}+\frac{F_{\mathrm{t}}}{m} \\
\dot{Q}=\left(-\frac{d \epsilon_{\mathrm{d}}+g}{R \epsilon_{0} \epsilon_{\mathrm{d}} \tilde{A}} Q-\frac{d \sigma_{\mathrm{d}}}{R \epsilon_{0} \epsilon_{\mathrm{d}}}\right) \cdot \frac{A_{0}}{A_{0}-\tilde{A}}
\end{array}\right.
$$

where $\omega$ and $\omega_{0}$ are the angular frequency of external acceleration and angular resonant frequency of the the spring-mass system, respectively; $\xi_{\mathrm{m}}$ is the mechanical damping factor, and $\tilde{A}(x)$ is the effective overlap area found with the FEM 


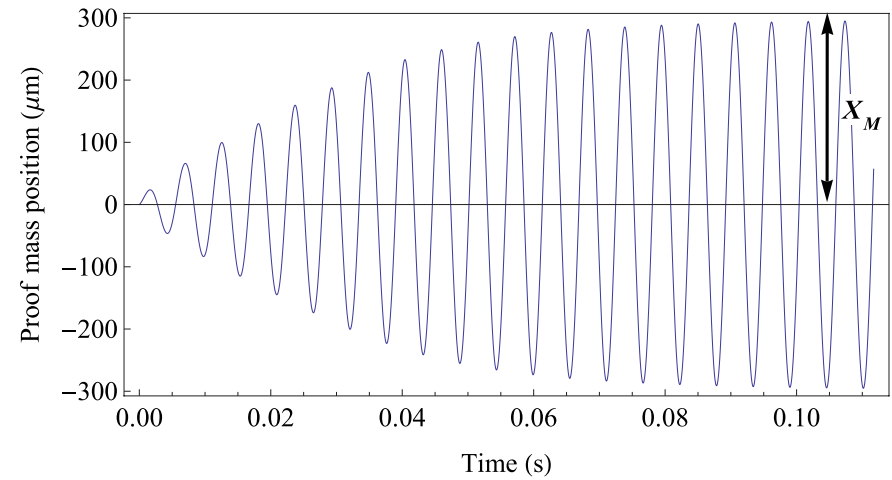

(a)

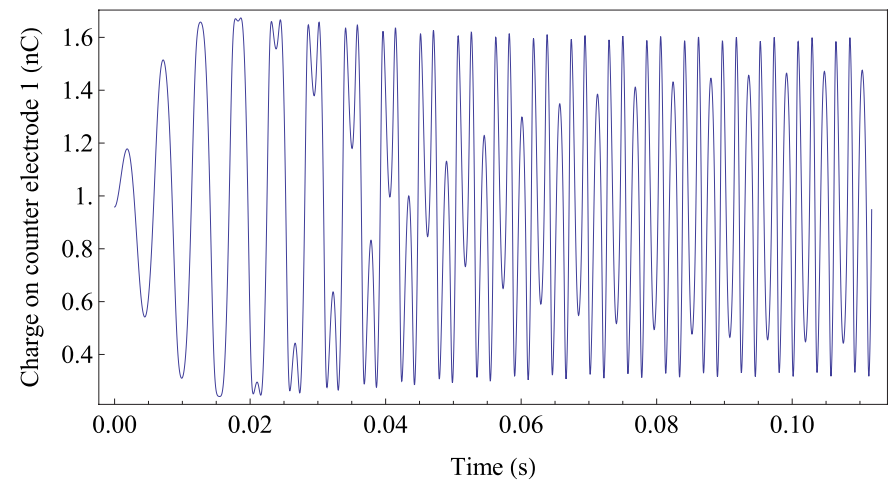

(b)

Fig. 7. Resonant solutions of equation set 19 for the first 20 oscillation periods. (a) $x(t)$. (b) $Q(t)$.

study (Eq. 13). $A_{0}$ has been redefined as equal to $A_{\mathrm{M}}+A_{\mathrm{m}}$ for consistency (when $\tilde{A}$ is equal to its mean value $\left(A_{\mathrm{M}}+A_{\mathrm{m}}\right) / 2$, then $\tilde{A}=A_{0}-\tilde{A}$ as it should, meaning that the electret is exactly in between two counter electrodes and overlaps equally with both). With this definition, the denominator of the second coupled equation does not go to zero at maximum or minimum overlap and therefore avoids the singularity in the current. A harmonic driving force at frequency $f$ and maximum acceleration $a_{0}$ has been used to model the vibration source. The quantities that couple the electrical and the mechanical domain are: (1) the transducer force, which depends on the electrical state of the system; (2) the overlap area (and hence the capacitance), which depends on the proof mass position.

Equation set 19 is solved numerically for $f=f_{0}=180 \mathrm{~Hz}$ (resonance condition) with the same parameters that were used to solve Eq. 11, when applicable. The remaining parameters are: $a_{0}=2.5 \mathrm{~m} / \mathrm{s}^{2}, m=6 \times 10^{-4} \mathrm{Kg}, \xi_{\mathrm{m}}=10^{-3}, \phi=\pi / 2$, $w=200 \mu \mathrm{m}$, and $R=100 \mathrm{M} \Omega$.

After an initial transient, the proof mass reaches a vibration amplitude of about $300 \mu \mathrm{m}$ (Fig. 7(a)), meaning that several electrodes are swept over during one period of oscillation. This is visually evident in Fig. 7(b) starting from $t=0.02 \mathrm{~s}$, when the amplitude reaches about $150 \mu \mathrm{m}$. Since $\phi=\pi / 2$, the electrode pattern displacement at rest is $125 \mu \mathrm{m}$ (Fig. 5). Then a vibration amplitude of $150 \mu \mathrm{m}$ allows the proof mass to travel just beyond the points of maximum and minimum overlap $\left(A_{\mathrm{M}}\right.$ and $\left.A_{\mathrm{m}}\right)$ which are also the points of maximum and minimum short-circuit charge. Beyond those points, the current

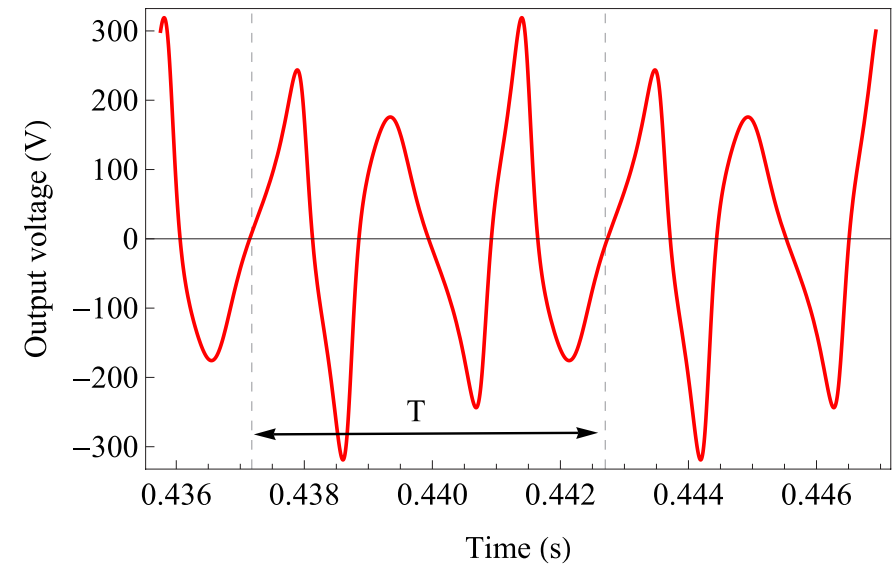

Fig. 8. Output voltage under highly non-linear conditions. The waveform cannot be approximated by a pure sine function.

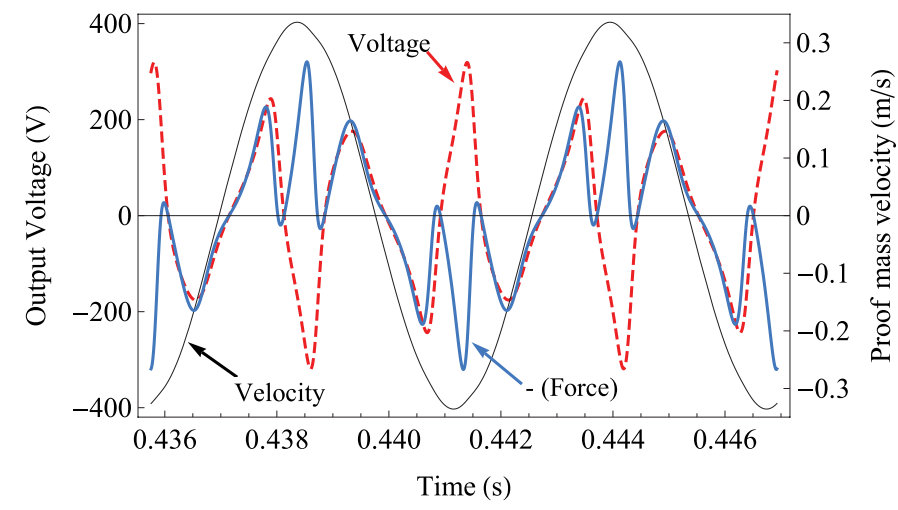

Fig. 9. Normalized transducer force in comparison with proof mass velocity and output voltage.

changes sign: this effect is seen in the notches appearing in Fig. 7(b) starting from $t=0.02 \mathrm{~s}$. Peano et al. [23] proved that for a similar electret-based harvester [24], the predicted output power can be wrong by two orders of magnitude if a linear model is employed under highly non-linear conditions. The same authors showed that a non-linear regime is also reached when a good choice of parameters leads to a high energy conversion efficiency (for instance, at $R \simeq R_{\mathrm{opt}}$ ). In this case $Q(t)$ is delayed and distorted with respect to $x(t)$ [as seen in Fig. 3(b)].

From Fig. 7(b), it can be observed that the charge oscillation amplitude slightly decreases after the first few periods. This can be understood by the following argument: as the mechanical vibration approaches its steady-state amplitude, $A(t)$ changes more and more rapidly. Therefore, a decreasing amount of charge flows between counter electrodes due to the fixed response time of the device equivalent circuit. Since response time increases with load resistance, a similar effect is also responsible for the decrease in output power when $R>R_{\text {opt }}$.

The steady-state output voltage is plotted in Fig. 8: the fundamental frequency of output voltage from the device is three times larger than the vibration frequency $f$ and the signal shape is not a simple sine function. Fig. 9 shows normalized plots of proof mass velocity, output voltage, and 


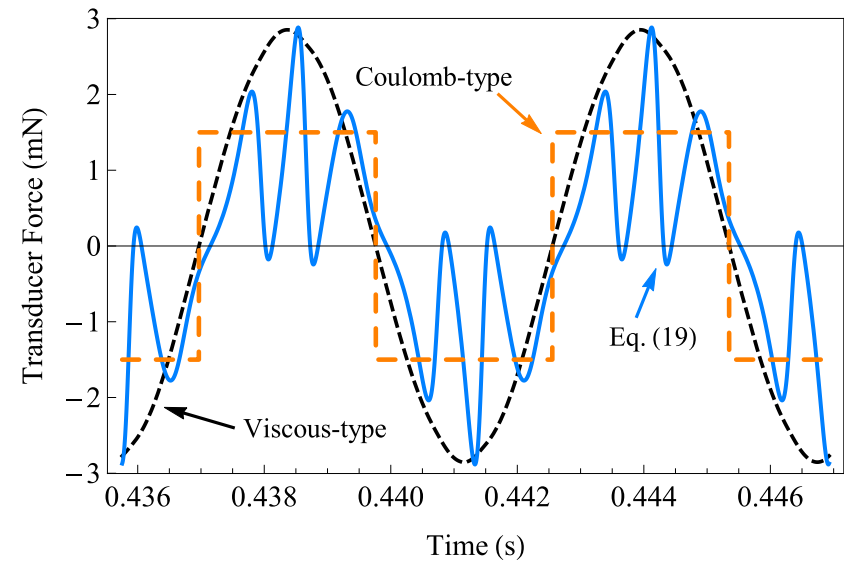

Fig. 10. Transducer force obtained by solving Eqs. 19, compared with hypothetical viscous-type and Coulomb-type damping forces.

transducer force. The sign of the force is almost always opposite to the velocity of the proof mass. It is thus a damping force but, as shown in Fig. 10, it cannot be modeled by either a viscous force (Eq. 2) or a Coulomb force (Eq. 3).

A viscous force approximation can, however, be appropriate under certain conditions. For a maximum vibration amplitude $X_{\mathrm{M}}$ less than $100 \mu \mathrm{m}$, the short circuit charge and effective area functions $Q^{s c}(x)$ and $\tilde{A}(x)$ could be linearized around the rest position $x_{0}=125 \mu \mathrm{m}$. Then $\tilde{A}(x)$ is proportional to $x$ and thus $\tilde{A}(t)$ is a pure sinusoid: the situation is qualitatively similar to the one in Fig. 3. Unless the load is very high, charge is proportional to position with a small phase lag [Fig. 3(a)] and thus current is proportional to velocity. For a coupled system the transducer force $F_{\mathrm{t}}$ defined by Eq. 18 is now just proportional to the voltage $V_{\mathrm{t}}$ because $\mathrm{d} Q^{s c} / \mathrm{d} x$ is constant and the term proportional to $\mathrm{d} C / \mathrm{d} x$ is small, again unless the load is very high. For a resistive load voltage is proportional to current, so it can be concluded that $F_{\mathrm{t}} \propto-\mathrm{d} x / \mathrm{d} t$ to a good approximation and Eqs. 1-2 are valid.

However, much of the work on electret-based energy harvesting so far has utilized narrow electrets compared to the maximum proof mass displacement [11], [13], [25] as a way to increase the output current. In such transducers several periods of the $Q^{s c}(x)$ function are covered within the displacement range so linearization is not possible. Eq. 1 is thus expected to be not as accurate as in systems where the viscous or Coulomb approximation are valid.

Fig. 11 shows the energy conversion loop for one oscillation period. Multiple sub-loops arise because of the periodic electrode structure: each counter electrode pair that is overlapped by an electret gives rise to a sub-loop. The average harvested power $P$ can be found by calculating the integral of the loop (harvested energy) and dividing by the oscillation period $T$. In our example, the power is $P \simeq 230 \mu \mathrm{W}$.

\section{EXPERIMENTAL VALIDATION FROM TwO CASE STUDIES}

\section{A. Device A (Crovetto et al.)}

An ESS energy harvesting device presented in [14] was designed to harvest energy from two perpendicular

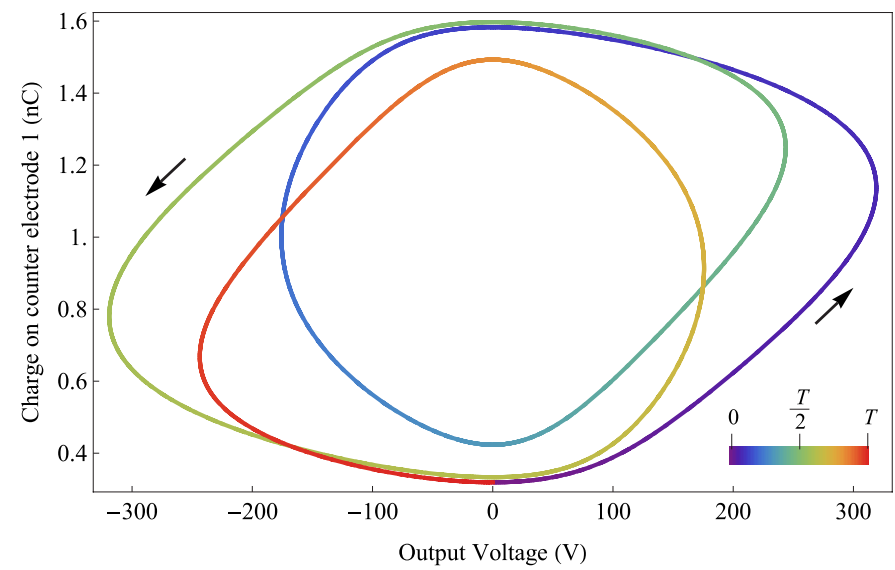

Fig. 11. Energy conversion loop for one vibration period of the proof mass. There are multiple sub-loops due to the periodic electret arrangement.

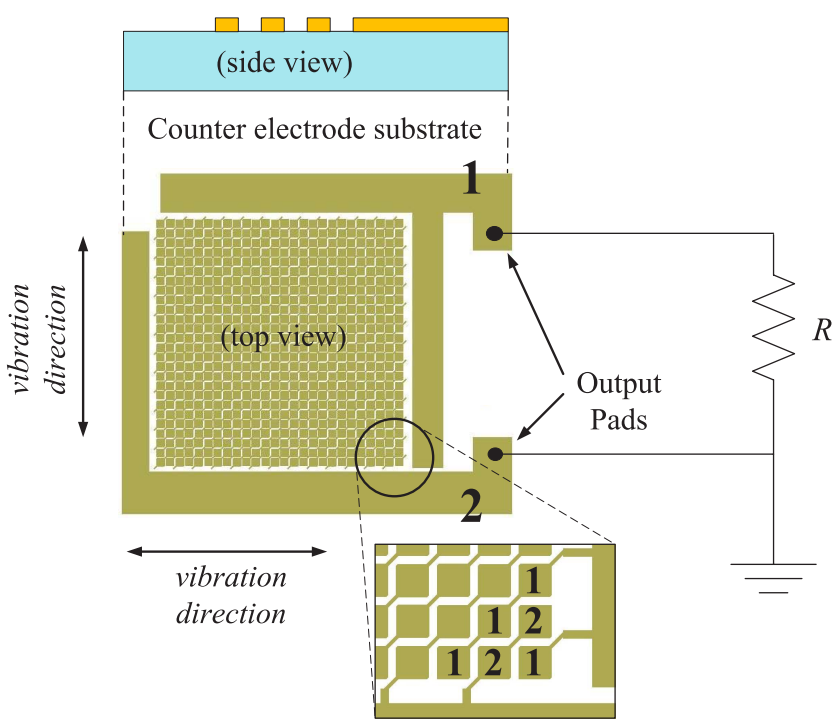

Fig. 12. Top view of the counter electrode plane for Device A. All type-1 counter electrodes are connected to output pad 1. All type-2 counter electrodes are connected to output pad 2. This allows energy harvesting from two perpendicular vibration directions.

vibration directions. To achieve this goal, counter electrodes of the same type (1 and 2) are connected diagonally as shown in Fig. 12. The parameter with the highest degree of uncertainty for Device $A$ is the mechanical damping factor $\xi_{\mathrm{m}}$. To obtain a good estimate, a modeled output is searched at a low load $R \ll R_{\text {opt }}$, where mechanical damping is dominant with respect to transducer damping. Firstly, all parameters (including the acceleration $a_{0}$ ) are set to their known or estimated values (Table I). Subsequently, $\xi_{\mathrm{m}}$ is fine-tuned in order to yield a steady-state displacement amplitude corresponding to the maximum allowed by the device spring system $\left(X_{\mathrm{M}}=230 \mu \mathrm{m}\right)$. The results from this procedure is $\xi_{\mathrm{m}}=7 \times 10^{-4}$, corresponding to a mechanical Q-factor $Q_{\mathrm{m}}=1 / 2 \xi_{\mathrm{m}} \simeq 700$. An excellent agreement between the model and the measurement has been achieved with this method (Fig. 13). The two secondary peaks around the main signal peak are due to a non-linearity of the system (the same electret overlaps with 4 counter electrodes during one 
This article has been accepted for inclusion in a future issue of this journal. Content is final as presented, with the exception of pagination.

TABLE I

PARAMETERS OF DeVice A

\begin{tabular}{llllll}
\hline$g$ & $100 \mu \mathrm{m}$ & $\varepsilon_{\mathrm{d}}$ & 2 & $m$ & $0.06 \mathrm{gram}$ \\
$d$ & $10 \mu \mathrm{m}$ & $\sigma_{\mathrm{d}}$ & $-0.16 \mathrm{mC} / \mathrm{m}^{2}$ & $A_{0}$ & $18 \mathrm{~mm}^{2}$ \\
$w$ & $200 \mu \mathrm{m}$ & $a_{0}$ & $0.04 \mathrm{~g}$ & $X_{\mathrm{M}}$ & $230 \mu \mathrm{m}$ \\
$l$ & $200 \mu \mathrm{m}$ & $f_{0}$ & $179 \mathrm{~Hz}$ & $\xi_{\mathrm{m}}$ & $7 \times 10^{-4}$ \\
$s$ & $50 \mu \mathrm{m}$ & $f$ & $179 \mathrm{~Hz}$ & $\phi$ & $\pi / 3$ \\
\hline
\end{tabular}

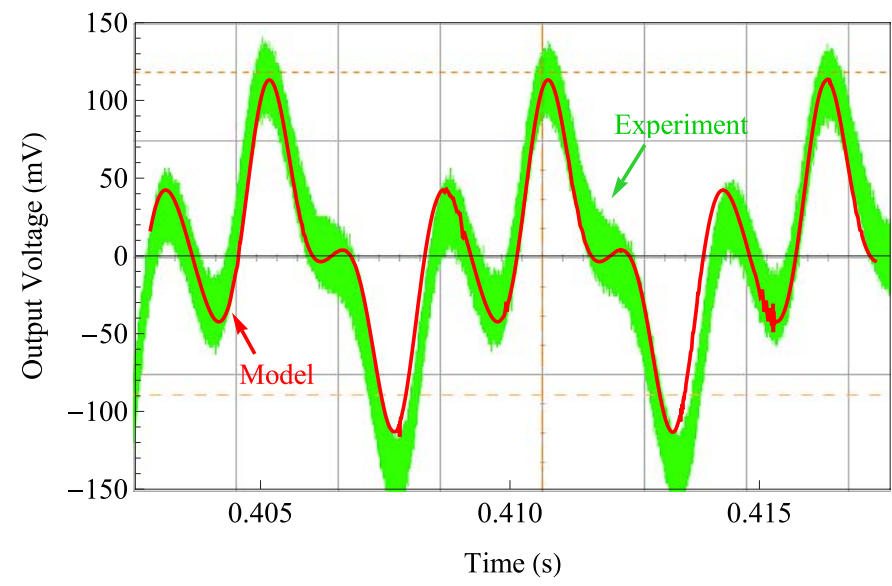

Fig. 13. Model prediction and experimental output voltage signal for Device A $\left(R=1 \mathrm{M} \Omega \ll R_{\text {opt }}\right)$. Approximately two and a half vibration periods are shown. The signal is not symmetrical because of the odd initial pattern overlap $\phi=\pi / 7$.

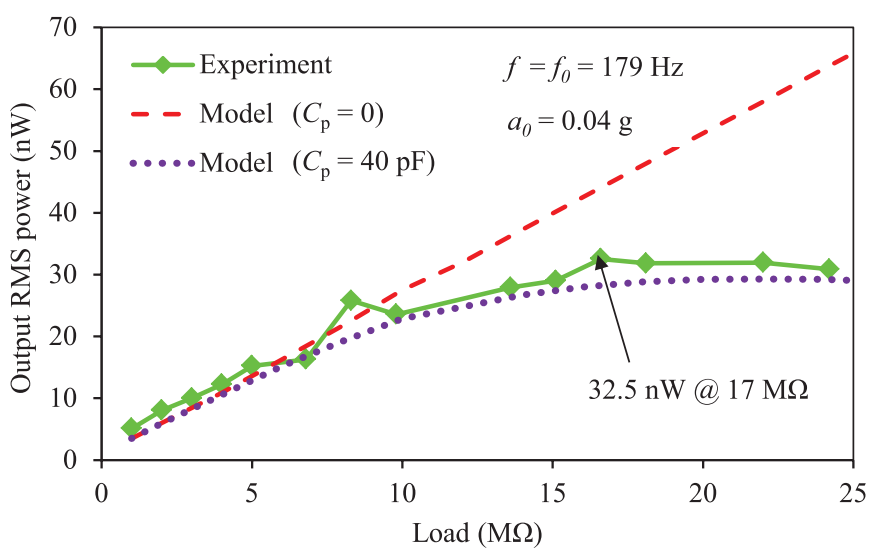

Fig. 14. Model prediction and experimental output power versus load for Device A. The parasitic capacitance is estimated to $40 \mathrm{pF}$.

oscillation period). The secondary peaks are not symmetric because of the odd initial pattern overlap $(\phi=\pi / 7)$.

Out model provides a very good estimate on the energy output for the low load range (Fig. 14). For higher load than $10 \mathrm{M} \Omega$, the parasitic capacitance will affect the measurement and the model should be further refined by adding a parasitic capacitor $C_{\mathrm{p}}$ in parallel with the load resistance [19]. Eq. 11 should be revised from $\mathrm{d} Q / \mathrm{d} t=V_{\mathrm{t}} / R$ to $\mathrm{d} Q / \mathrm{d} t=V_{\mathrm{t}} / R+$ $C_{\mathrm{p}} \mathrm{d} V_{\mathrm{t}} / \mathrm{d} t$. Under a sinusoidal signal approximation the output power is approximately

$$
P_{\mathrm{out}, \mathrm{p}} \simeq \frac{P_{\mathrm{out}, 0}}{1+\left(\omega C_{\mathrm{p}} R\right)^{2}}
$$

where $P_{\text {out, } 0}$ is the predicted output power assuming no parasitic capacitance (dashed line in Fig. 14) and $\omega$ is the angular frequency of the output signal. If $\omega \ll 1 / R C_{\mathrm{p}}$ the effect of the parasitic capacitance is negligible; if $\omega \gg 1 / R C_{\mathrm{p}}$ almost no power is harvested; if $\omega \simeq 1 / R C_{\mathrm{p}}$ output power is reduced by one half. With a parasitic capacitance of $40 \mathrm{pF}$ and assuming $\omega$ as roughly equal to the vibration angular frequency, we have achieved a good agreement between model and experiment (dotted line in Fig. 14).

\section{B. Device B (Naruse et al.)}

The single-sided device presented in [13] can harvest energy in one dimension. The authors propose a stripe masked electret structure with the full silicon substrate acting as a base electrode. Our model is applicable to this device because the silicon substrate is electrically isolated from the counter electrodes.

The operating conditions of Device B are quite different from those of Device A (Table II). The key differences are the following: (1) most of the data are taken in a non-resonant regime where $f=2 \mathrm{~Hz}$ and $f_{0}=4 \mathrm{~Hz}$; (2) the proof mass collides with the device frames when $a_{0}=0.4 \mathrm{~g}$; (3) the maximum displacement amplitude is very large with respect to the electret width, so up to 60 counter electrodes can be swept by the proof mass over one period. The height $d$ of the $\mathrm{SiO}_{2}$ electrets is not provided by the authors. It has been estimated to $2 \mu \mathrm{m}$ since it is close to the upper practical thickness for thermally-grown $\mathrm{SiO}_{2}$ films. The mass of the proof mass $m$ is not provided explicitly but can be derived from equation 1 using the theoretical maximum power calculated in [13] for $a_{0}=0.1 \mathrm{~g}$. In addition, the surface charge density is calculated from the surface potential shown in the paper.

Fig. 15 shows the output power for the whole relevant load spectrum. The model fits well with the experimental data for $R<R_{\text {opt }}$ but it predicts a slightly higher optimal load than the one found experimentally. This might be due to the unknown parasitic capacitances and an underestimated mechanical damping $\xi_{\mathrm{m}}$. Despite of the uncertain issues, our model still gives a much better estimate of the power output comparing to the unrealistic value (55 times higher than the measurement) from the theoretical calculation.

\section{Validation Outcome}

Our model has been tested in two very different scenarios for ESS devices. Device A is designed for $2 \mathrm{D}$ vibrations and it has a relatively small $X_{\mathrm{M}} / w$ ratio with an odd initial 
TABLE II

Parameters of DeVice B

\begin{tabular}{llllll}
\hline$g$ & $48 \mu \mathrm{m}$ & $\varepsilon_{\mathrm{d}}$ & 3.9 & $m$ & 5.8 gram \\
$d$ & $2 \mu \mathrm{m}$ & $\sigma_{\mathrm{d}}$ & $-10.4 \mathrm{mC} / \mathrm{m}^{2}$ & $A_{0}$ & $75 \mathrm{~mm}^{2}$ \\
$w$ & $200 \mu \mathrm{m}$ & $a_{0}$ & $0.4 \mathrm{~g}$ & $X_{\mathrm{M}}$ & $7.5 \mathrm{~mm}$ \\
$l$ & $10 \mathrm{~mm}$ & $f_{0}$ & $4 \mathrm{~Hz}$ & $\xi_{\mathrm{m}}$ & N.A. \\
$s$ & $50 \mu \mathrm{m}$ & $f$ & $2 \mathrm{~Hz}$ & $\phi$ & 0 \\
\hline
\end{tabular}

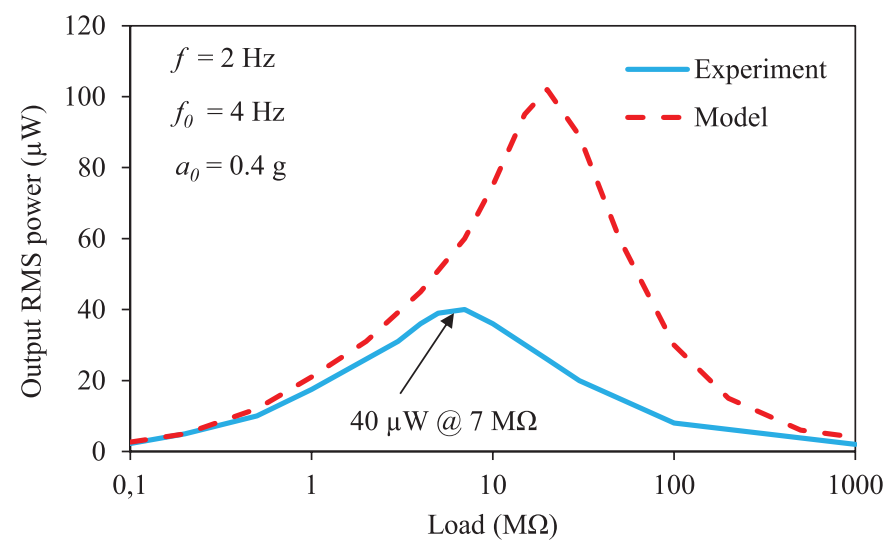

Fig. 15. Model prediction and experimental output power versus load for Device B (experimental data taken from [13]).

pattern overlap. Its base electrodes are separated from one another, and it has been operated at resonance, with low acceleration and high frequency. Conversely, Device B has a typical electrode arrangement for $1 \mathrm{D}$ vibrations, a large $X_{\mathrm{M}} / w$ ratio, the whole silicon substrate as a base electrode, and it has been operated off resonance, with high acceleration and low frequency. Therefore, our model is expected to perform well on ESS energy harvesting devices with various electrode geometries, provided that the actual $\tilde{A}(x)$ is simulated with a FEM study. Parasitic capacitance and more complex load circuits can be simulated with some modification of the model equations, as shown in Section VI.

\section{PARAMETER OPtimization}

For a realistic energy harvesting application, the choice of harvester parameters needs to be adjusted according to the expected acceleration and frequency of the vibration source. The acceleration amplitude $a_{0}$ and frequency $f$ should therefore be regarded as fixed constraints. Additionally, the active area of the device is likely to have an upper limit in the design phase due to size constraints. Hence the side length of the harvester $L$ is also pre-determined.

Sizing and charge biasing of the device capacitances (parameters $\left.\sigma_{\mathrm{d}}, \epsilon_{\mathrm{d}}, d, g, w, s\right)$ requires a type of optimization that is highly dependent on technical matters. For instance: (1) the maximum $\sigma_{\mathrm{d}}$ obtainable by corona charging is an increasing function of electrode width $w$ and it depends on the electret material (parameter $\epsilon_{\mathrm{d}}$ ); (2) the air gap $g$ must be large enough to avoid or minimize the risk of snapping between the proof mass and the counter electrode substrate to a minimum; (3) the electrode spacing $s$ must be tuned to find an optimal balance between effective electrode area (Eq. 13), parasitic capacitances between counter electrodes, and the risk of short circuits. For the purpose of our study this set of parameters will be taken from Table II, which is a promising technical achievement.

The proof mass is assumed to be made of silicon, due to its dominant use in mass-produced MEMS devices. Its thickness $h$ is realistically constrained to be smaller than $h_{\text {max }}=1 \mathrm{~mm}$ to prevent unwanted complexity in microfabrication. Similarly, the external load $R$ is limited in the upper range $\left(R_{\max }=10 \mathrm{M} \Omega\right)$ due to a reduced usability of the output signal at very high load resistances and the increased influence of parasitic capacitances. $\phi$ is taken as zero for simplicity.

Given such assumptions, it is possible to find the optimal displacement limit $X_{\mathrm{M}}$, and therefore the optimal length of the proof mass, for a fixed total area $L^{2}$ (Fig. 16(a)). The result can be plotted at different vibration accelerations (or at different vibration frequencies), to find the maximum power that can be harvested under any configuration of the vibration source. Fig. 17 shows the optimization algorithm in detail and Fig. 18 shows the resulting output power against $X_{M}$ and $a_{0}$.

When no mechanical damping is applied $\left(\xi_{\mathrm{m}}=0\right)$, the optimal $X_{\mathrm{M}}$ is found to be $L / 4$ for the whole acceleration range of our study. This means that power is maximized when the proof mass consumes half of the available space. This result confirms the existing prediction by Mitcheson et al. [21]. Because the load resistance is limited to $10 \mathrm{M} \Omega$ during the simulation, the maximum achievable power saturates with acceleration (Fig. 19 and Table III). More power can be harvested at high acceleration only if the upper limitation on $R$ is increased (or, alternatively, if a better combination of capacitive parameters is realized).

If mechanical damping is present $\left(\xi_{\mathrm{m}}=5 \times 10^{-4}\right.$, that is a Q-factor of 1000) the situation changes as follows. At low acceleration the vibration amplitude might never reach the optimal $L / 4$ regardless of how small the load resistance is, because mechanical damping may be the dominant amplitudelimiting factor. For example, with a Q-factor of 1000 and $a_{0}=0.05 \mathrm{~g}$, maximum power is obtained when $X_{\mathrm{M}}=L / 10$ (Table IV). In a higher acceleration range, the main difference from the no-damping case is that higher acceleration is required to achieve the same performance (Fig. 19).

With the current parameters, the maximum power that theoretically can be harvested by an ESS device is $17 \mu \mathrm{W}$ at $0.01 \mathrm{~g}$, 


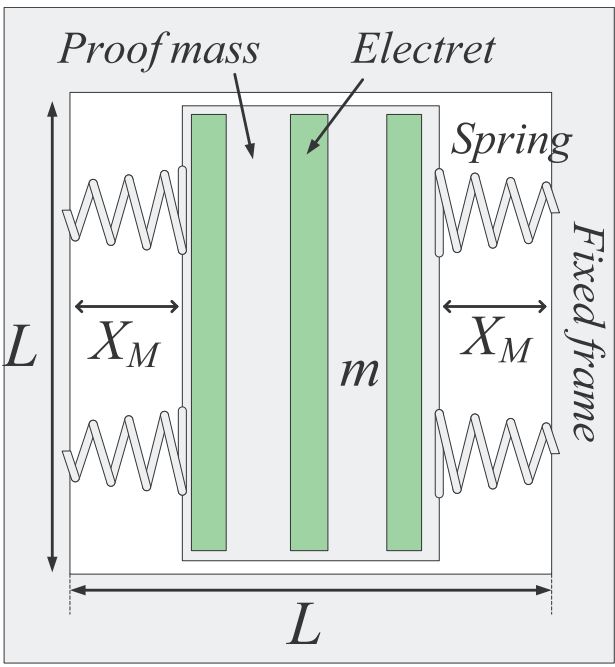

(a)

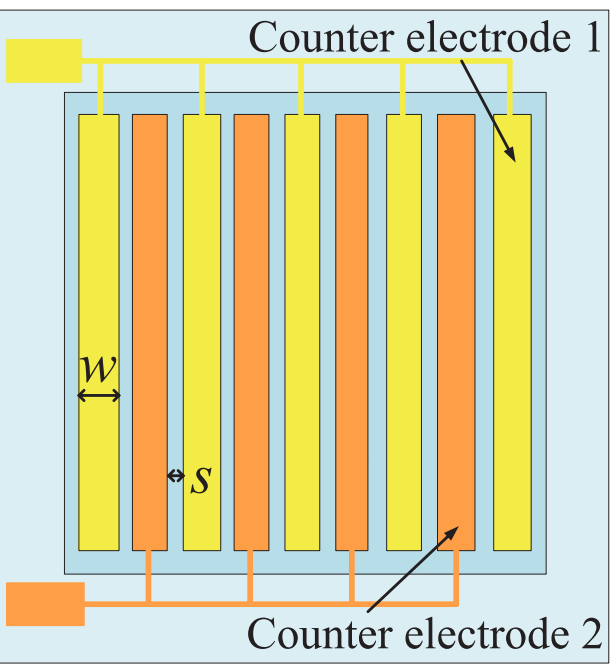

(b)

Fig. 16. Sample device used for optimization. The active area is a square with a side length of $L$. (a) Electret substrate. (b) Counter electrode substrate.

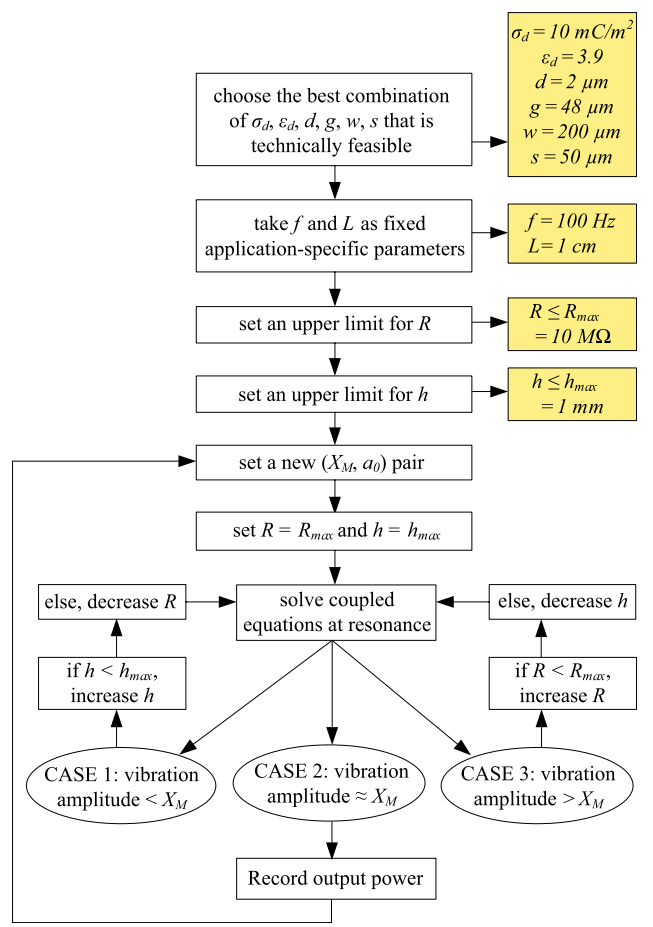

Fig. 17. Optimization algorithm used to find the maximum power achievable by each $\left(X_{\mathrm{M}}, a_{0}\right)$ pair within the range of our study. The yellow boxes indicate the fixed parameters and constraints.

$46 \mu \mathrm{W}$ at $0.05 \mathrm{~g}, 95 \mu \mathrm{W}$ at $0.1 \mathrm{~g}, 464 \mu \mathrm{W}$ at $0.5 \mathrm{~g}$, and $549 \mu \mathrm{W}$ from $0.6 \mathrm{~g}$ onwards. Without collisions, Device B in [13] could have harvested $232 \mu \mathrm{W}$ at its resonance frequency of $4 \mathrm{~Hz}$, at an acceleration of $0.4 \mathrm{~g}$ and with a load resistance $R=10 M \Omega$, just by setting the proof mass length to its optimal value $L / 2$, and its thickness to $h=950 \mu \mathrm{m}$. Therefore Device B achieved only $19 \%$ of its optimal output power.

\section{POWER CONDITIONING}

Typical raw output signals of ESS energy harvesters (Figs. 8 and 13, and [13]) are not suitable for powering

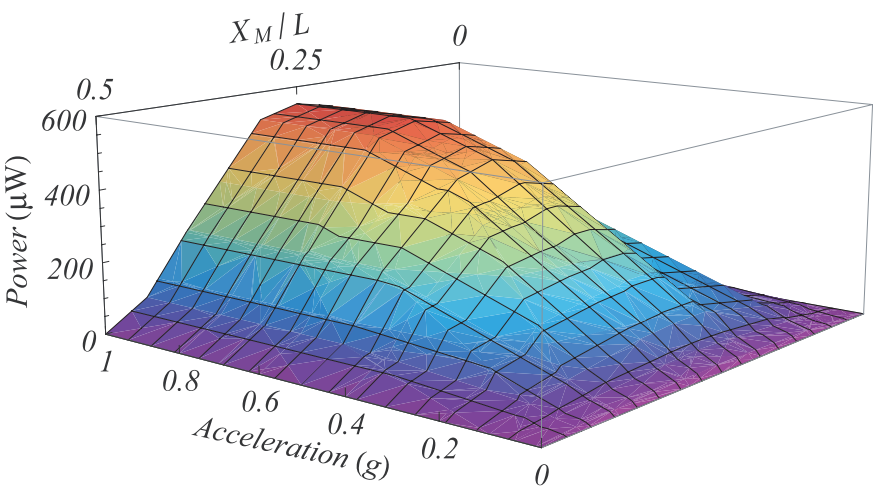

Fig. 18. Output power optimization with no mechanical damping $\left(\xi_{\mathrm{m}}=0\right)$. Power is maximized when $X_{\mathrm{M}}=L / 4$ at all accelerations.

practical devices. The waveform can be irregular and, in the case of many electret-to-electrode overlaps $\left(w \ll X_{\mathrm{M}}\right)$, it is also amplitude-modulated by the proof mass velocity. The optimal load of electrostatic generators is usually too high for most applications [7]-[14] and a DC power supply is typically needed instead of AC. Therefore the signal must be conditioned by a non-linear electrical circuit in order to match the input requirements of the powered device [26]. In this section we discuss the effect of two variants of a standard power conditioning circuit on a few key quantities, such as transducer current $\mathrm{d} Q / \mathrm{d} t$, transducer voltage $V_{\mathrm{t}}$, transducer force $F_{\mathrm{t}}$, output voltage $V_{\mathrm{o}}$, output power, and optimal load.

The signal-conditioning circuit is shown in Fig. 20: the two electrodes of the energy harvester are connected to a full-wave bridge rectifier delivering the resulting rectified signal to a storage capacitor $C_{\mathrm{s}}$ and, through a switch, to the external load. We will use a sample ESS device with a high $X_{\mathrm{M}} / w$ ratio (extremely non-linear), source acceleration $a_{0}=0.25 \mathrm{~g}$ and frequency $f=100 \mathrm{~Hz}$. The diodes' forward voltage drop is set to $0.7 \mathrm{~V}$ (as in typical silicon diodes).

We first examine the case when the switch is always closed (continuous system), similarly to the circuit that was used to 
TABLE III

Optimization Results With No Mechanical Damping

\begin{tabular}{lllll}
\hline$a_{0}(\mathrm{~g})$ & $R_{\mathrm{opt}}(\mathrm{M} \Omega)$ & $\mathrm{h}(\mathrm{mm})$ & optimal $X_{\mathrm{M}} / L$ & $P_{\text {out }}(\mu \mathrm{W})$ \\
\hline 0.01 & 0.3 & 1 & 0.25 & 17 \\
0.05 & 0.8 & 1 & 0.25 & 46 \\
0.1 & 1.7 & 1 & 0.25 & 95 \\
0.25 & 4 & 1 & 0.25 & 231 \\
0.5 & 8 & 1 & 0.25 & 464 \\
0.75 & 10 & 0.8 & 0.25 & 549 \\
1 & 10 & 0.6 & 0.25 & 549 \\
\hline
\end{tabular}

TABLE IV

Optimization Results With $\xi_{\mathrm{m}}=5 \times 10^{-4}$ (Mechanical Q-FaCtoR of 1000)

\begin{tabular}{lllll}
\hline$a_{0}(\mathrm{~g})$ & $R_{\text {opt }}(\mathrm{M} \Omega)$ & $\mathrm{h}(\mathrm{mm})$ & optimal $X_{\mathrm{M}} / L$ & $P_{\text {out }}(\mu \mathrm{W})$ \\
\hline 0.01 & 0.2 & 1 & 0.02 & 1.2 \\
0.05 & 0.2 & 1 & 0.1 & 5.5 \\
0.1 & 0.2 & 1 & 0.25 & 11 \\
0.25 & 2.3 & 1 & 0.25 & 129 \\
0.5 & 6.5 & 1 & 0.25 & 369 \\
0.75 & 10 & 0.95 & 0.25 & 549 \\
1 & 10 & 0.7 & 0.25 & 549 \\
\hline
\end{tabular}

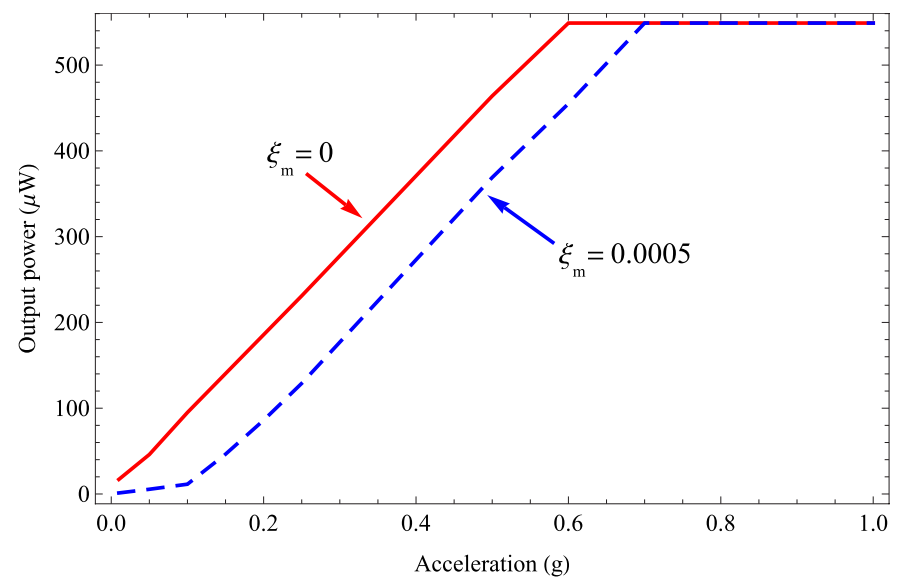

Fig. 19. Optimized output power versus source acceleration at the optimal $X_{\mathrm{M}} / L$ ratio for each acceleration. The case of no mechanical damping and the case of a mechanical damping factor $\xi_{\mathrm{m}}=5 \times 10^{-4}$ are considered. Power saturates at high acceleration due to the constraint on $R$.

test Device B [13]. Two different operational states should be distinguished: (1) the conductive state, when current flows through either pair of diodes, and (2) the non-conductive state, triggered at the moment when $\mathrm{d} Q / \mathrm{d} t=0$. In the nonconductive state, the current is prevented from changing sign instantly because that would result in an instant change of the transducer voltage $V_{\mathrm{t}}$ from $V_{\mathrm{o}}+2 V_{\mathrm{d}}$ to $-\left(V_{\mathrm{o}}+2 V_{\mathrm{d}}\right)$. But $V_{\mathrm{t}}$ ultimately depends on the surface charge densities of each electrode (Eqs. 9-10), and they can change either through a current flow or through a change in the overlap area $\tilde{A}$ due to a proof mass position change. A current flow is impossible, though, because it would occur through the same pair of diodes that were conducting before the switch, thus violating Kirchoff's voltage law. Therefore we conclude that the current must be zero during the transition of $V_{\mathrm{t}}$ from $V_{\mathrm{o}}+2 V_{\mathrm{d}}$ to $-\left(V_{\mathrm{o}}+2 V_{\mathrm{d}}\right)$. The transition is due to a change in the proof mass position $x$ and it is not instantaneous.

With these premises, Eq. 8 for the purely resistive case must be replaced by two new equations for each state of the system. For the conductive state:

$$
\left|\frac{\mathrm{d} Q(t)}{\mathrm{d} t}\right|=\frac{V_{\mathrm{o}}(t)}{R}+C_{\mathrm{s}} \frac{\mathrm{d} V_{\mathrm{o}}(t)}{\mathrm{d} t}
$$

as Kirchoff's current law, and

$$
V_{\mathrm{o}}(t)=\left|V_{\mathrm{t}}(t)\right|-2 V_{\mathrm{d}}
$$

as Kirchoff's voltage law. In the non-conductive state the smoothing capacitor and the load resistance form an independent RC discharging circuit so the equations become:

$$
V_{\mathrm{o}}(t)=-R C_{\mathrm{s}} \frac{\mathrm{d} V_{\mathrm{o}}(t)}{\mathrm{d} t}
$$

and

$$
\frac{\mathrm{d} Q(t)}{\mathrm{d} t}=0
$$

The transducer force expression is still given by Eq. 18 because the transducer itself is unchanged.

Our model predictions are shown in Figs. 21, 22, and 23, where the smoothing capacitor has been set to $C_{\mathrm{s}}=10 \mathrm{nF}$. 


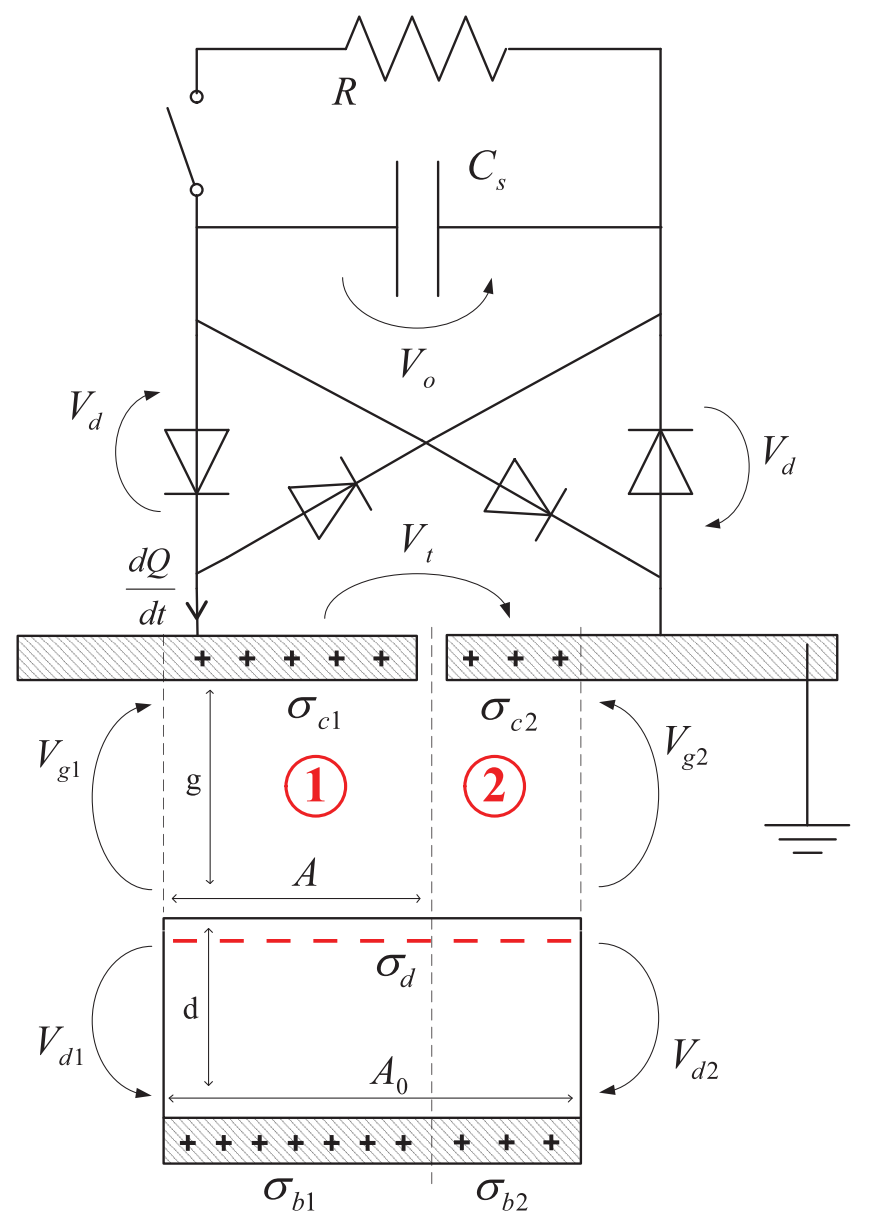

Fig. 20. ESS energy harvesting device connected to a power-conditioning circuit including a full-wave bridge rectifier, a smoothing or storage capacitor, and a switch to periodically discharge the capacitor through the load.

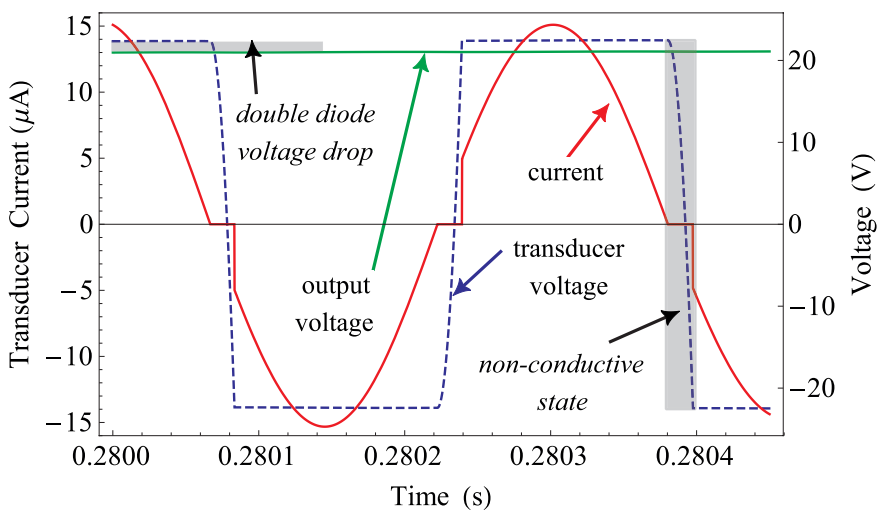

Fig. 21. Conductive and non-conductive states in a continuous rectified system. While the current is zero, the transducer voltage $V_{\mathrm{t}}$ ramps from from $V_{\mathrm{o}}+2 V_{\mathrm{d}}$ to $-\left(V_{\mathrm{o}}+2 V_{\mathrm{d}}\right)$ or viceversa. The time range is $1 / 20$ of a vibration period of the proof mass.

In Fig. 21 the behavior of system in the conductive and non-conductive states is evident: when the current is zero, the transducer voltage ramps up or down until its absolute value reaches $V_{\mathrm{o}}+2 V_{\mathrm{d}}$. At the optimal load of $3.4 \mathrm{M} \Omega$ the output voltage $\left|V_{\mathrm{t}}\right|$ is rectified to about $21 \mathrm{~V}$ with a ripple amplitude of about $1.4 \%$ (Figs. 22 and 23). The average output power after $t=0.15 \mathrm{~s}$ is $131 \mu \mathrm{W}$. The transducer force $F_{\mathrm{t}}$ oscillates at twice the frequency of the transducer voltage

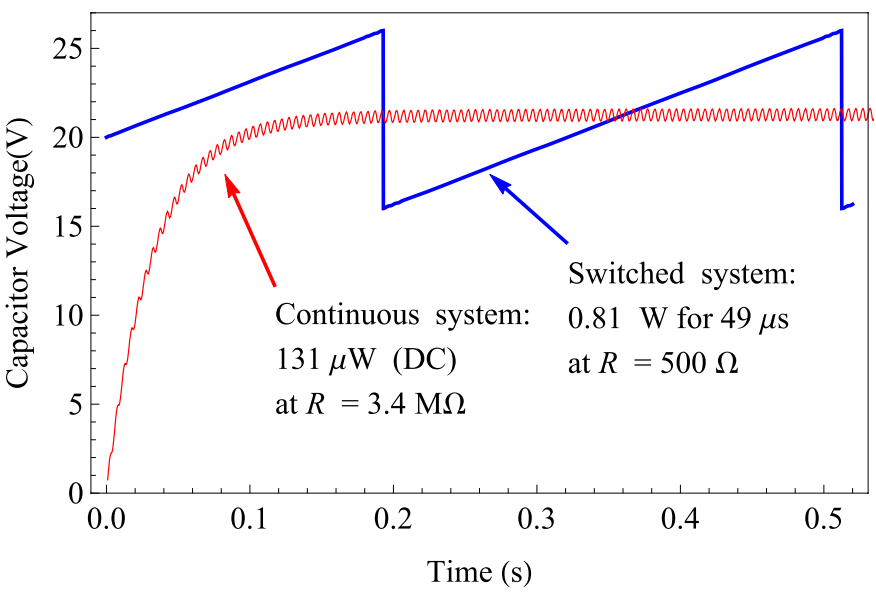

Fig. 22. Voltage across the smoothing (or charging) capacitor $C_{\mathrm{s}}$ at the optimal load for the continuous and switched systems over 50 proof mass vibration periods. The voltage range of the switched system is designed to be near the optimal voltage of the continuous system.

$V_{\mathrm{t}}$ but its amplitude is approximately constant (following the small ripple voltage oscillations) and its sign is opposite to the velocity of the proof mass. It was stated in [25] that, for a similar EBS harvester, this force has the form of a square wave and thus the device behaves like a Coulomb damper under the effect of the present circuit. However, for a device with a high $X_{\mathrm{M}} / w$ ratio, this is true only if one disregards the higher-frequency oscillations of $F_{\mathrm{t}}$ within the squares.

Then we turn to the case of a voltage-controlled switch, similar to the one in [25]. The switch is first opened to charge up the capacitor until $\left|V_{\mathrm{o}}\right|=V_{\max }$. When this upper threshold is reached the switch is closed to allow the capacitor to discharge through the load. The switch is reopened when $\left|V_{\mathrm{o}}\right|=V_{\min }$ to let the capacitor charge again to $V_{\max }$. We use the same equations as for the continuous case (Eqs. 2124) with a discharge capacitor $C_{\mathrm{s}}=200 \mathrm{nF}$ and a small load $R=500 \Omega$ which could be a realistic input impedance for a powered device. We set $V_{\max }=26 \mathrm{~V}$ and $V_{\min }=16 \mathrm{~V}$ to let the harvester operate at optimal damping close to its optimal output voltage (see Fig. 22). Apart from the slow voltage ramp followed by rapid discharge, the waveforms are similar to the continuous case (Fig. 24). The ripple has been further reduced due to the higher capacitance. Every $320 \mathrm{~ms}$, about $40 \mu \mathrm{J}$ is harvested in $49 \mu \mathrm{s}$ (Figs. 22 and 24), corresponding to an average burst power of $0.86 \mathrm{~W}$ and a time-averaged harvested power of $124 \mu \mathrm{W}$. That is $94.7 \%$ of the power obtained with the continuous system: this small discrepancy occurs because the transducer does not operate at its optimal voltage all the time. Hence the vibration amplitude of the proof mass is not always equal to its maximum displacement limit $X_{\mathrm{M}}$, but the average voltage cannot be lowered further because the proof mass would start colliding with the device frames. To get even closer to the theoretical power limit, $C_{\mathrm{s}}$ or the $V_{\max }-V_{\min }$ interval can be decreased. However, both actions will result in an even shorter power delivery time to the load, so the most appropriate design will depend on the application. In any case, setting $V_{\max }$ and $V_{\min }$ so that the device operates near its optimal voltage is a key element in designing the conditioning circuit. 

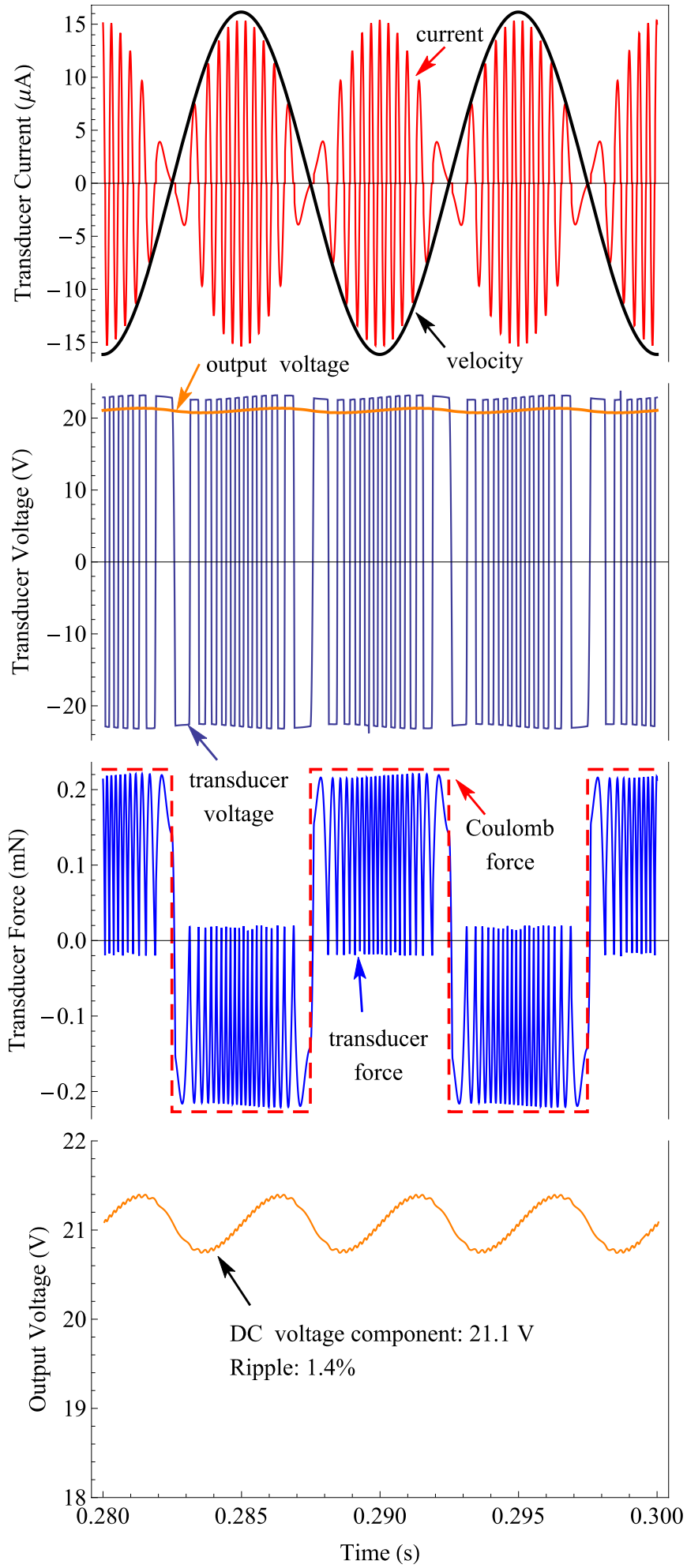

Fig. 23. Time trace of transducer current $\mathrm{d} Q / \mathrm{d} t$, transducer voltage $V_{\mathrm{t}}$, transducer force $F_{\mathrm{t}}$, and output voltage $V_{\mathrm{o}}$ for a continuous rectified system over two proof mass vibration periods. The current is modulated by the velocity of the proof mass $\mathrm{d} x / \mathrm{d} t$. The output voltage and the absolute value of the transducer voltage differ by two diode voltage drops $(1.4 \mathrm{~V})$. The transducer force is compared to a hypothetical Coulomb force. The nonconductive states can barely be seen at this resolution.

If yet the same ESS device is operated with a purely resistive load, the resulting output signals and force are shown in Fig. 25. The amplitude of the transducer force is proportional
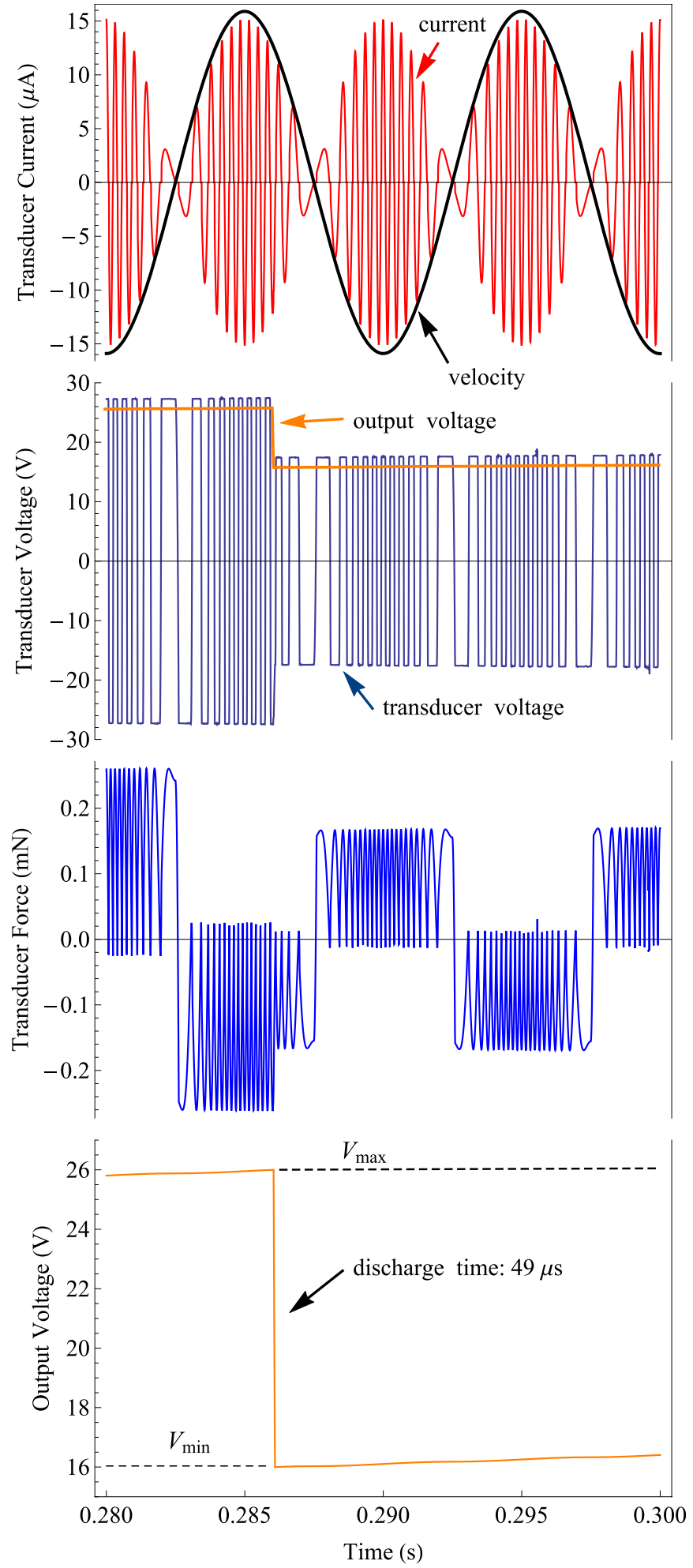

Fig. 24. Time trace of transducer current $\mathrm{d} Q / \mathrm{d} t$, transducer voltage $V_{\mathrm{t}}$, transducer force $F_{\mathrm{t}}$, and output capacitor voltage $V_{\mathrm{o}}$ for a switched rectified system. The output voltage and the absolute value of the transducer voltage differ by two diode voltage drops $(1.4 \mathrm{~V})$. Both transducer voltage and transducer force are affected by the switch. The current is mostly unaffected because the vibration amplitude only changes slightly at the time of the switch, due to operation near the optimal voltage. The non-conductive states can barely be seen at this resolution.

to velocity but $F_{\mathrm{t}}$ cannot be regarded as a viscous force because it oscillates at twice the frequency of the voltage while being modulated by the velocity. The average output power 

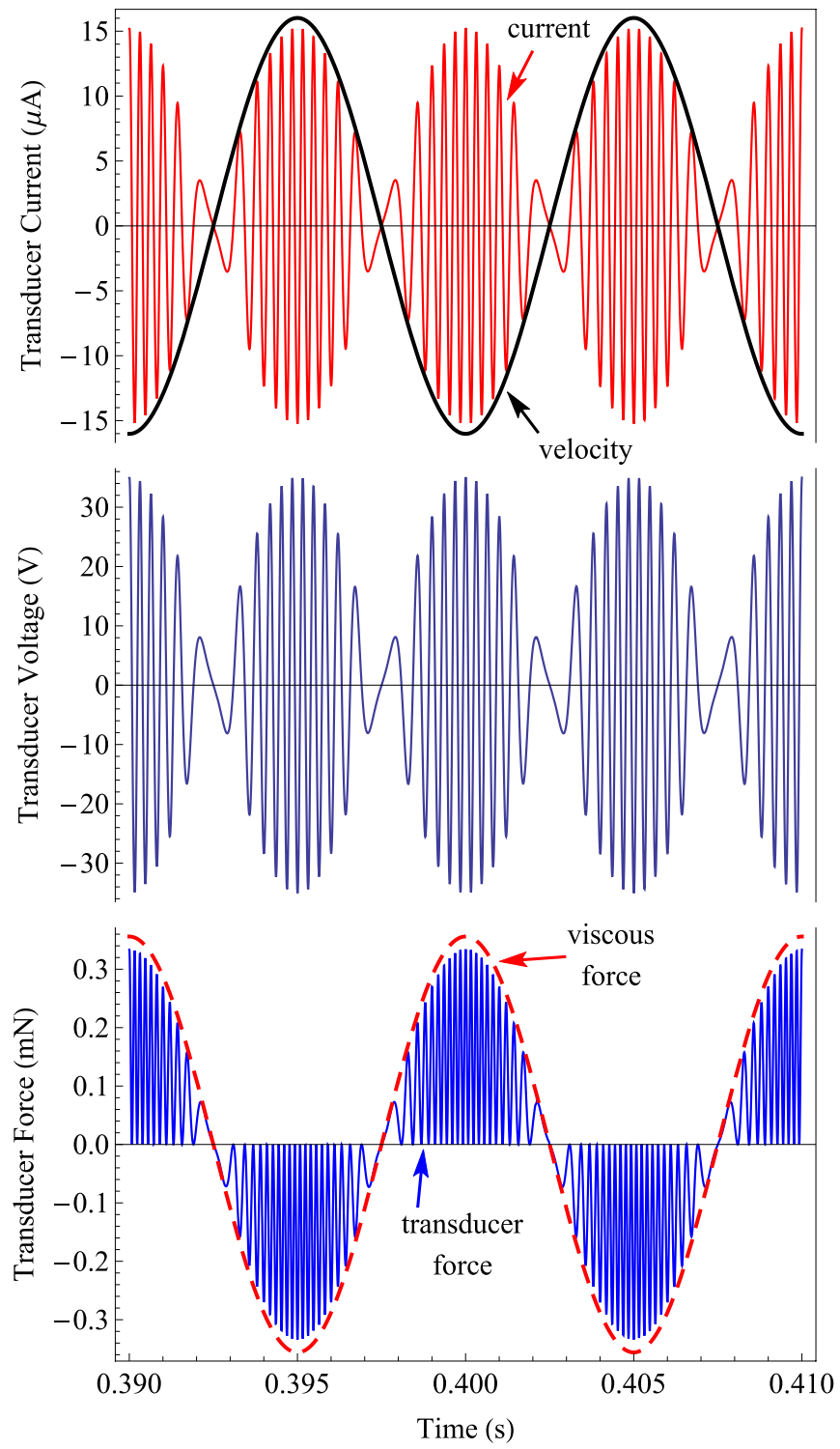

Fig. 25. Time trace of transducer current $\mathrm{d} Q / \mathrm{d} t$, transducer voltage $V_{\mathrm{t}}$, and transducer force $F_{\mathrm{t}}$ with a purely resistive load. Here the transducer voltage equals the output voltage. The transducer force is compared to a hypothetical viscous force.

is $134 \mu \mathrm{W}$ at an optimal load of $2.3 \mathrm{M} \Omega$, a very similar result to the rectified continuous system. The harvested power is slightly higher because there are no losses associated with diodes.

\section{CONCLUSION}

In this paper, we have proposed a new model for in-plane oscillating electrostatic energy harvesting devices. It turns out that the ESS design can generate more power than EBS design with the same external load thanks to its faster response time. From both analytical expression and numerical calculation, we have shown that the electrostatic transduction force is neither a viscous damping force which is proportional to the velocity of the proof mass nor a Coulomb-type constant damping force. Instead of those types of damping force, we have shown that the transduction force is more related to the output voltage.
With an FEM simulation, the fringe effect of the capacitor is studied. An effective overlap area is defined and well fitted by a sinusoidal function. From the coupled electro-mechanical model, the output voltage is numerically calculated. We have verified this model with the parameters from one of our devices fabricated previously. A good level of agreement is found for both the power output and the signal waveform. The model is further applied with the parameters from a reference, where a good estimate of power output is obtained. Using this model, we have further optimized the design where maximum power output is achieved when the maximum displacement of the proof mass is set to a quarter of the chip's side length. With the optimal design, power output up to $549 \mu \mathrm{W}$ could be possibly harvested for a $1 \mathrm{~cm}^{2}$ chip from an external acceleration of $0.6 \mathrm{~g}$. The effect of a power-conditioning circuit has been investigated: power outputs comparable to the purely resistive load case have been obtained and guidelines have been found to keep the output power near its maximum for a switched system. This study indicates that the electrostatic energy harvesting devices can provide as high power output as piezoelectric or electromagnetic devices and thus have promising applications for future wireless sensor networks.

\section{REFERENCES}

[1] S. P. Beeby, M. J. Tudor, and N. M. White, "Energy harvesting vibration sources for microsystems applications," Meas. Sci. Technol., vol. 17, no. 12, pp. R175-R195, 2006.

[2] P. Mitcheson, E. Yeatman, G. Rao, A. Holmes, and T. Green, "Energy harvesting from human and machine motion for wireless electronic devices," Proc. IEEE, vol. 96, no. 9, pp. 1457-1486, Sep. 2008.

[3] R. Xu, A. Lei, C. Dahl-Petersen, K. Hansen, M. Guizzetti, K. Birkelund, et al., "Screen printed PZT/PZT thick film bimorph MEMS cantilever device for vibration energy harvesting," Sens. Actuators A, Phys., vol. 188, pp. 383-388, Dec. 2012.

[4] S. Roundy and P. K. Wright, "A piezoelectric vibration based generator for wireless electronics," Smart Mater. Struct., vol. 13, no. 5, pp. 1131-1142, 2004

[5] H. Kulah and K. Najafi, "An electromagnetic micro power generator for low-frequency environmental vibrations," in Proc. 17th IEEE Int. Conf. MEMS, Jan. 2004, pp. 237-240.

[6] P. Glynne-Jones, M. Tudor, S. Beeby, and N. White, "An electromagnetic vibration-powered generator for intelligent sensor systems," Sens. Actuators A, Phys., vol. 110, nos. 1-3, pp. 344-349, 2004.

[7] Y. Suzuki, "Recent progress in MEMS electret generator for energy harvesting," IEEJ Trans. Electr. Electron. Eng., vol. 6, no. 2, pp. 101-111, Mar. 2011.

[8] A. Paracha, P. Basset, D. Galayko, F. Marty, and T. Bourouina, "A silicon MEMS DC/DC converter for autonomous vibration-to-electrical-energy scavenger," IEEE Electron Device Lett., vol. 30, no. 5, pp. 481-483, May 2009.

[9] Y. Sakane, Y. Suzuki, and N. Kasagi, "The development of a highperformance perfluorinated polymer electret and its application to micro power generation," J. Micromech. Microeng., vol. 18, no. 10, pp. 104011-1-104011-6, 2008.

[10] S. Boisseau, G. Despesse, T. Ricart, E. Defay, and A. Sylvestre, "Cantilever-based electret energy harvesters," Smart Mater. Struct., vol. 20, no. 1, p. 105013, Oct. 2011.

[11] T. Sterken, P. Fiorini, G. Altena, C. Van Hoof, and R. Puers, "Harvesting energy from vibrations by a micromachined electret generator," in Proc. Int. Conf. Solid-State Sens., Actuators, Microsyst., Jun. 2007, pp. 129 32.

[12] H. wen Lo and Y.-C. Tai, "Parylene-based electret power generators," J. Micromech. Microeng., vol. 18, no. 10, pp. 104006-1-104006-8, 2008.

[13] Y. Naruse, N. Matsubara, K. Mabuchi, M. Izumi, and S. Suzuki, "Electrostatic micro power generation from low-frequency vibration such as human motion," J. Micromech. Microeng., vol. 19, no. 9, pp. 094002-1-094002-5, 2009. 
[14] A. Crovetto, F. Wang, and O. Hansen, "An electret-based energy harvesting device with a wafer-level fabrication process," J. Micromech. Microeng., vol. 23, no. 11, pp. 114010-1-114010-10, 2013.

[15] F. Wang and O. Hansen, "Invisible surface charge pattern on inorganic electrets," IEEE Electron Device Lett., vol. 34, no. 8, pp. 1047-1049, Aug. 2013.

[16] F. Wang and O. Hansen, "Electrostatic energy harvesting device with out-of-the-plane gap closing scheme," in Proc. 17th Int. Conf. SolidState Sens., Actuators, Microsyst., Jun. 2013, pp. 2237-2240.

[17] J. Boland, Y.-H. Chao, Y. Suzuki, and Y. Tai, "Micro electret power generator," in Proc. IEEE 16th Annu. Int. Conf. Micro Electro Mech. Syst., Jan. 2003, pp. 538-541.

[18] S. Boisseau, G. Despesse, and A. Sylvestre, "Optimization of an electretbased energy harvester," Smart Mater. Struct., vol. 19, no. 7, p. 075015, 2010.

[19] U. Bartsch, "Electret-based resonant micro energy harvesting in two dimensions," Ph.D. dissertation, Dept. Microsyst. Eng., Univ. Freiburg, Freiburg im Breisgau, Germany, 2010.

[20] C. Williams and R. Yates, "Analysis of a micro-electric generator for microsystems," Sens. Actuators A, Phys., vol. 52, nos. 1-3, pp. 8-11, Mar. 1996.

[21] P. Mitcheson, T. Green, E. Yeatman, and A. Holmes, "Architectures for vibration-driven micropower generators," J. Microelectromech. Syst., vol. 13, no. 3, pp. 429-440, Jun. 2004.

[22] W. T. Thomson, Theory of Vibration with Applications, 3rd ed. Englewood Cliffs, NJ, USA: Prentice-Hall, 1988.

[23] F. Peano and T. Tambosso, "Design and optimization of a MEMS electret-based capacitive energy scavenger," J. Microelectromech. Syst., vol. 14, no. 3, pp. 429-35, 2005.

[24] R. P. T. Sterken, K. Baert, and S. Borghs, "Power extraction form ambient vibration," in Proc. 3rd Workshop Semicond. Sens. Actuators, Nov. 2002, pp. 680-683.

[25] D. Miki, Y. Suzuki, and N. Kasagi, "Effect of nonlinear external circuit on electrostatic damping force of micro electret generator," in Proc. Int. Solid-State Sens., Actuators Microsyst. Conf., Jun. 2009, pp. 636-639.

[26] L. R. Clare and S. G. Burrow, "Power conditioning for energy harvesting," Proc. SPIE, vol. 6928, pp. 69280A-1-69280A-13, Apr. 2008.

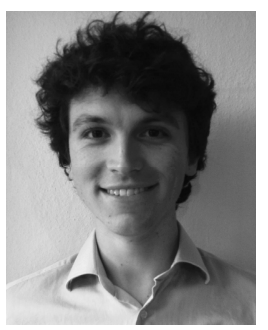

Andrea Crovetto received the M.Sc. degree in physics engineering and nanotechnology from Politecnico di Milano, Italy, in 2012. His studies included a project on electret-based energy harvesting at the Technical University of Denmark. He is currently pursuing the $\mathrm{Ph}$.D. degree in thin-film solar cell technology at the Technical University of Denmark. His research interests include electromechanical transducers, photovoltaics, microfabrication, and modeling of semiconductor devices.

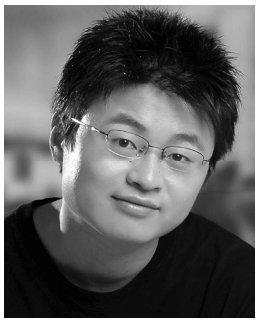

Fei Wang (S'06-M'09-SM'12) received the B.S. degree in mechanical engineering from the University of Science and Technology of China, Hefei, China, in 2003, and the Ph.D. degree in microelectronics from Shanghai Institute of Microsystem and Information Technology, Chinese Academy of Science, Shanghai, China, in 2008.

He was a Postdoctoral Researcher with the Department of Micro- and Nanotechnology, Technical University of Denmark, where he had been promoted to Assistant Professor in 2010. Since 2013, he has been an Associate Professor in the Department of Electronic and Electrical Engineering, South University of Science and Technology of China. His current research interests include micro energy harvesting, MEMS and NEMS devices, IC, and semiconductor testing.

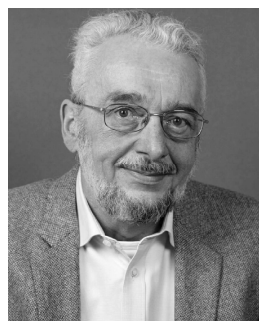

Ole Hansen received the M.Sc. degree in microtechnology from the Technical University of Denmark (DTU), Lyngby, Denmark, in 1977.

Since 1977 he has worked with micro-and nanotechnology and applications of the technology within electronics, metrology, sensing, catalysis and energy harvesting. He is Professor at DTU Nanotech, the Technical University of Denmark, where he is heading the Silicon Microtechnology group, with activities within lithography based micro- and nanotechnology. Current research interests include sustainable energy, photocatalysis and tools for characterizing catalytic processes. Since 2005 he has been part of the Danish National Research Foundation Center CINF, Center for Individual Nanoparticle Functionality. 